\title{
Metal-Binding Properties of Human Centrin-2 Determined by Micro-Electrospray Ionization Mass Spectrometry and UV Spectroscopy
}

\author{
Theodore A. Craig
}

Department of Medicine, Mayo Clinic College of Medicine and Mayo Clinic and Foundation, Rochester, Minnesota, USA

\section{Linda M. Benson and H. Robert Bergen III}

Mayo Proteomics Research Center, Mayo Clinic College of Medicine and Mayo Clinic and Foundation, Rochester, Minnesota, USA

\section{Sergei Y. Venyaminov* and Jeffrey L. Salisbury}

Department of Biochemistry and Molecular Biology, Mayo Clinic College of Medicine and Mayo Clinic and Foundation, Rochester, Minnesota, USA

\section{Zachary C. Ryan and James R. Thompson}

Department of Physiology and Biomedical Engineering, Mayo Clinic College of Medicine and Mayo Clinic and Foundation, Rochester, Minnesota, USA

\section{Justin Sperry and Michael L. Gross}

Department of Chemistry, Resource for Biomedical Mass Spectrometry, Washington University in St. Louis, St. Louis, Missouri, USA

\section{Rajiv Kumar $^{+}$}

Department of Medicine, Mayo Clinic College of Medicine and Mayo Clinic and Foundation, Rochester, Minnesota, USA

We analyzed the metal-binding properties of human centrin-2 (HsCen-2) and followed the changes in HsCen-2 structure upon metal-binding using micro-electrospray ionization mass spectrometry ( $\mu$ ESI-MS). Apo-HsCen-2 is mostly monomeric. The ESI spectra of HsCen-2 show two charge-state distributions, representing two conformations of the protein. HsCen-2 binds four moles calcium/mol protein: one mol of calcium with high affinity, one additional mol of calcium with lower affinity, and two moles of calcium at low affinity sites. HsCen-2 binds four moles of magnesium/mol protein. The conformation giving the lower charge-state HsCen-2 by ESI, binds calcium and magnesium more readily than does the higher charge-state HsCen-2. Both conformations of HsCen-2 bind calcium more readily than magnesium. Calcium was more effective in displacing magnesium bound to HsCen-2 than vice versa. Binding of a peptide from a known binding partner, the xeroderma pigmentosum complementation group protein C (XPC), to apo-HsCen-2, occurs in the presence or the absence of calcium. Near and far-UV CD spectra of HsCen-2 show little difference with addition of calcium or magnesium. Minor changes in secondary structure are noted. Melting curves derived from temperature dependence of molar ellipticity at $222 \mathrm{~nm}$ for HsCen-2 show that calcium increases protein stability whereas magnesium does not. $\Delta 25 \mathrm{HsCen}-2$ behaves similarly to HsCen-2. We conclude that HsCen-2 binds calcium and magnesium and that calcium modulates HsCen-2 structure and function by increasing its stability without undergoing significant changes in secondary or tertiary structure. (J Am Soc Mass Spectrom 2006, 17, 1158-1171) (c) 2006 American Society for Mass Spectrometry

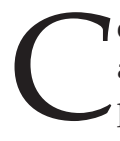
entrins (or caltractins) are small $(\sim 20 \mathrm{KDa})$, acidic EF-hand proteins that are important components of the centriole [1-5]. Centrin was first

Published online June 5, 2006

Address reprint requests to Dr. R. Kumar, Mayo Clinic, 200 First St. SW, Rochester, MN 55905, USA. E-mail: rkumar@mayo.edu

* Also at the Department of Molecular Pharmacology and Experimental Therapeutics, Mayo Clinic College of Medicine and Mayo Clinic and Foundation, Rochester, MN 55905.

+ Also at the Department of Biochemistry and Molecular Biology, Mayo Clinic College of Medicine and Mayo Clinic and Foundation, Rochester, MN 55905. discovered in the flagellar apparatus of the green alga, Tetraselmis striata, where it functions as a calcium-sensing regulator of centriole structure and function, and as part of the calcium-dependent contractile apparatus [6]. Centrins have been identified in numerous higher and lower organisms including fungi (e.g., Dictyostelium), plants, and mammals, where they function in centrosome duplication, localization, and segregation [7]. Centrins control cilia-regulated $\mathrm{Ca}^{2+}$ channels [8], mammalian cilia function, and could potentially play a role in the pathogenesis of 
various renal cystic diseases [9]. $\mathrm{Ca}^{2+}$-triggered assembly of a centrin-transducin complex in the vertebrate visual system may regulate transducin translocation through the photoreceptor connecting cilium [10-12]. Centrins also play a role in microtubule severing through interactions with Sfi1 $[13,14]$.

Centrin-2 (also known as caltractin-1), an important component of the centriolar apparatus, is an EF-hand protein that is essential for the function of the spindle pole apparatus and centrosome duplication [5]. Centrin-2 is found in both cytoplasmic and nuclear compartments and is a component of nearly all xeroderma pigmentosum (XP) nucleotide excision repair complexes, where it binds and stabilizes the XPC protein [5, 15-18].

Full length human centrin-2 (HsCen-2), and the carboxyl-terminal domains of HsCen-2 fragments, bind calcium at one high affinity site, probably in the most carboxyl-terminal EF-hand [19, 20]. Previous studies suggested that HsCen-2 is dimeric and binds only two calciums per dimer, likely to site IV in each monomer [19]. Studies of an N-terminal 25 amino acid truncation of HsCen-2 suggest that the $\mathrm{N}$-terminus is involved in the calcium-dependent dimerization of the protein [21]. On the other hand, Veeraraghavan et al. showed that Chlamydomonas centrin-2 binds $4 \mathrm{~mol}$ of calcium $/ \mathrm{mol}$ protein [22].

Electrospray ionization mass spectrometry (ESI-MS) has emerged as a powerful tool with which to study the conformation, dynamics, and metal-binding properties of proteins [23-40]. The mass measurement of intact noncovalent complexes, for example, can be used to determine ligand stoichiometry as well as relative affinity constants [41, 42]. Furthermore, the charge-state distributions of proteins seen in an ESI mass spectrum contain information on the different conformers present in solution under the conditions of the spray [43-45]. In general, native conformers have charge-state distributions that are more narrow and at higher $m / z$ than those distributions of partially or fully unfolded conformers. Thus, the parameters that affect protein conformation in solution (e.g., $\mathrm{pH}$, temperature, and solution composition) can be varied and the consequences measured by ESI-MS. The changes in charge-state distributions mirror the changes in solution of one conformer in the presence of another.

In parallel with these applications, Kaltashov and coworkers [46] advanced this approach by quantifying conformation distributions using chemometrics. Their approach deconvolved overlapping charge states to afford the various conformer distributions in solution. In addition to quantifying distributions, there is also an opportunity to separate conformations (in terms of $\mathrm{m} / \mathrm{z}$ ) and measure, for example, the binding property of one conformer with respect to that of another. Ray and coworkers [47] took this latter approach and demonstrated that the native conformer of pfTIM binds more ligand (ANS, a fluorescent dye) than the partially unfolded conformers represented by higher charge states.
Considering the role HsCen-2 has in several important biological functions and disease states, we wished to take advantage of the sensitivity and speed of ESI-MS to determine the properties of HsCen-2. Given that this protein is conformationally flexible [19-21], we probed if its flexibility might be manifest by multiple chargestate distributions in its ESI mass spectrum. Additionally, the ESI-MS approach would allow us to determine, in a facile manner, the association of other proteins with HsCen-2. We asked whether HsCen-2 exists in more than one conformational state, and if so, whether the calcium and magnesium-ion stoichiometry and affinity were different for each conformer. We also investigated whether the binding of calcium and magnesium are associated with marked changes in the secondary structure, as revealed by optical spectroscopy, and in the melting temperature of the protein.

\section{Experimental}

\section{General}

Protein amino-terminal and DNA sequencing were carried out using automated methods as described [48-53]. Oligonucleotide synthesis was performed using an Applied Biosystems DNA/oligonucleotide synthesizer [54, 55] (Applied Biosystems, Foster City, CA). UV spectra of proteins and nucleic acids were recorded using a model DU-70 or DU-640 Beckman spectrophotometer (Beckman Instruments, Fullerton, CA). Protein concentrations were determined by the BioRad Protein Reagent (Bradford) method [56] (BioRad Laboratories, Hercules, CA) and the BCA Reagent with bovine serum albumin as standard (Pierce Biochemicals, Rockford, IL) and by measuring UV absorbance using the millimolar absorptivity of the HsCen-2 at $277 \mathrm{~nm}$ of $\mathrm{E}_{\mathrm{mM}} \sim 1.46$ $\mathrm{mM}^{-1} \mathrm{~cm}^{-1}$. SDS and non-denaturing PAGE were carried out using a PhastGel apparatus (Amersham Biosciences Corp., Piscataway, NJ) and precast gels. Reagents were from Sigma (St. Louis, MO) unless otherwise indicated.

\section{Biosynthesis of Human Centrin-2 and $\Delta 25$ Human Centrin-2 Proteins}

Full-length HsCen-2 and $\Delta 25$ HsCen-2 proteins were biosynthesized in E. coli BL21 cells, transformed with pGEX-6P-1 plasmid (Amersham Biosciences Corp.) containing the HsCen-2 cDNA sequence (GenBank accession NM_004344) using PCR and procedures described previously [23, 24, 32, 57-60]. The purified full length insert was ligated into BamHI- and XhoI-treated pGEX6P-1 plasmid. The $\Delta 25 \mathrm{HsCen}-2$ sequence, derived from PCR using a 5' primer with an additional PreScission protease site incorporated, was inserted into the pCR2.1 TOPO vector (Invitrogen Corp., Carlsbad, CA). Plasmid isolated from transformed E. coli TOP10 cells was cleaved with EcoRI. The purified $\Delta 25 \mathrm{HsCen}-2$ fragment was ligated into EcoRI cut pGEX-6P-1 plasmid and used 
to transform E. coli BL21 cells. Before expression, DNA sequencing of both strands of final pGEX-6P-1 HsCen-2 constructs was carried out and the expected DNA sequence for the constructs was verified. Transformed E. coli BL21 cells with HsCen-2 and $\Delta 25$ HsCen-2 pGEX-6P-1 constructs were grown in 2XYT medium at $37^{\circ} \mathrm{C}$ to an absorbance of 0.6 at $600 \mathrm{~nm}$. Protein expression was induced with $1.0 \mathrm{mM}$ isopropylthiogalactoside at $37^{\circ} \mathrm{C}$. After $4 \mathrm{~h}$, the cells were collected by centrifugation at $4000 \times g$ and lysed in lysis buffer (PBS pH 7.0 with $5 \mathrm{mM}$ EDTA, $5 \mathrm{mM}$ DTT, $0.1 \mathrm{mM}$ aminoethylbenzene sulfonyl fluoride $\mathrm{HCl}$ (AEBSF) (Bio-Research Products, Inc., North Liberty, IA) using 0.1-mm glass beads and a Bead Beater (Biospec Products, Bartlesville, $\mathrm{OK})$. The clarified lysate was applied to a column of glutathione-Sepharose resin (Clontech, Palo Alto, CA) and washed with $50 \mathrm{mM}$ Tris, $1 \mathrm{mM}$ EDTA, $1 \mathrm{mM}$ DTT, $\mathrm{pH} 7.4$ (WB). The fusion GST- HsCen-2 protein was eluted in WB containing $20 \mathrm{mM}$ glutathione (reduced), concentrated to a few $\mathrm{ml}$, and treated with PreScission protease (Amersham Biosciences Corp.) at $4{ }^{\circ} \mathrm{C}$. The protease-treated protein was purified by chromatography on a Mono-Q HR26/10 column (Amersham Biosciences Corp.). A NaCl gradient (0-1 M NaCl; $1 \mathrm{~L}$ total volume) was developed over $2 \mathrm{~h}$. Mono-Q fractions were assessed by Coomassie blue-stained SDS PAGE gels and fractions containing pure HsCen-2 or $\Delta 25$ HsCen-2 were pooled, concentrated and dialyzed against buffer ( $20 \mathrm{mM}$ Tris, $1 \mathrm{mM}$ DTT, $1 \mathrm{mM}$ EDTA, $\mathrm{pH}$ 7.4). This yielded, after PreScission protease cleavage, for full length HsCen-2 a construct with five amino acids (GPLGS) added to the N-terminus derived from the PreScission Protease cleavage site and the linker region containing the pGEX-6P-1 BamHI site, and for $\Delta 25 \mathrm{HsCen-2,}$ a protein construct lacking the first 25 amino acids in HsCen-2, with two amino acids (GP) added to the $\mathrm{N}$-terminus, derived from the additional PreScission protease recognition/cleavage sequence.

\section{Protein Identification}

Amino-terminal amino acid sequencing afforded HsCen-2 construct sequences that were identical to the sequences expected: for the full length $\mathrm{HsCen}-2$ constructs: GPLGS followed by amino acids 1-40 of HsCen-2; and for $\Delta 25$ HsCen-2, GP followed by amino acids 26-35 of HsCen-2. Analysis of full-length Centrin and $\Delta 25$ Centrin by $\mu$ ESI-MS afforded a multiply charged ion series, which on transformation revealed a single species of relative molecular mass of $20149 \mathrm{Da}$ (expected 20148Da) for the full length and a mass of $17,086 \mathrm{Da}$ (expected $17084 \mathrm{Da}$ ) for the $\Delta 25$ human centrin construct. This experimental value is well within the $0.01 \%$ accuracy for such measurements. Tryptic peptides derived from full length centrin were analyzed by LC-MS/MS. A tryptic peptide map was obtained covering $90 \%$ of the expected protein sequence. The biosynthetic proteins were thus unambiguously characterized by $\mu$ ESI-MS mass spectrometry,
N-terminal sequencing, and by tryptic digestion and peptide analysis by MS/MS to be the expected constructs of human centrin and $\Delta 25$ centrin.

\section{XPC Peptide Synthesis and Preparation}

The 17 amino acid human xeroderma pigmentosa group C peptide (NWKLLAKGLLIRERLKR) Asn847Arg863 (XPC) [16] was synthesized in the Mayo Proteomics Research Center Peptide Synthesis Facility. A stock solution $1.54 \mathrm{mg} / \mathrm{ml}(\sim 730 \mu \mathrm{M})$ (by weight) was made by dissolving XPC peptide into $20 \mathrm{mM}$ ammonium acetate, $10 \mu \mathrm{M}$ EDTA (free acid) $\mathrm{pH} 6.9$ and subsequently used for $\mu$ ESI-MS measurements.

\section{Microelectrospray Ionization Mass Spectrometry ( $\mu E S I-M S)$}

Before infusion into the $\mu$ ESI-MS apparatus, stock solutions $(72 \mu \mathrm{M})$ of purified HsCen-2 or $\Delta 25 \mathrm{HsCen}-2$ were prepared. Biosynthesized HsCen-2 or $\Delta 25$ HsCen-2 in $50 \mathrm{mM}$ Tris, $1 \mathrm{mM}$ sodium ethylene diamine tetraacetic acid (Na EDTA), $1 \mathrm{mM}$ DTT, pH 7.4, were dialyzed extensively against $20 \mathrm{mM}$ ammonium acetate, $10 \mu \mathrm{M}$ EDTA (free acid) $\mathrm{pH} 6.9$ at $4{ }^{\circ} \mathrm{C}$ in a $10 \times 0.5 \mathrm{ml}$ microdialyzer, 1000 MWCO SpectraPor 6 dialysis membrane (Spectrum Industries, Rancho Dominguez, CA). $10 \mu \mathrm{M}$ EDTA was present to scavenge divalent cations before metal titrations. Stock solutions were used for $\mu$ ESI-MS as described below.

$\mu$ ESI-MS analyses of HsCen-2 and the metal ion titration experiments were performed in positive mode on a Micromass LCT mass spectrometer (Waters Corp., Milford, MA) using a modified Z-spray $\mu$ electrospray source. The mass spectra were scanned from mass to charge $(\mathrm{m} / \mathrm{z}) 700-5000$ at a scan speed of $1.9 \mathrm{~s}$ and instrument resolution of 4000 . Vacuum pressure and sample cone, RF lens, and extraction cone voltages were optimized for detection of noncovalent interactions.

Working solutions of $17 \mu \mathrm{M}$ or $52 \mu \mathrm{M}$ HsCen 2 and $\Delta 25 \mathrm{HsCen}-2$ were prepared using $25 \mathrm{mM}$ ammonium acetate, $\mathrm{pH} 7.5$, from the $72 \mu \mathrm{M}$ stock solutions. Samples were introduced into the electrospray source by direct infusion at a flow rate of $0.3 \mu \mathrm{L} / \mathrm{min}$. Metal ion titration experiments were carried out by incubating Hs-Cen2 with appropriate concentrations of metal salts for $30 \mathrm{~min}$. Stock solutions of metal ions as the acetate salt were prepared in $25 \mathrm{mM}$ ammonium acetate, $\mathrm{pH}$ 7.5. The final $\mathrm{pH}$ of the incubation solutions ranged from 7.5 (no metals ions) to 7.4 (500 $\mu \mathrm{M}$ metal ions). In the competitive binding experiments, a secondary metal salt was added after the $30 \mathrm{~min}$ incubation and allowed to incubate for an additional $30 \mathrm{~min}$ before direct infusion of samples into the $\mu$ ESI source. Xeroderma pigmentosum group $\mathrm{C}$ peptide (XPC) was prepared as described above. HsCen-2 and XPC incubates were analyzed after $30 \mathrm{~min}, 6 \mathrm{~h}$, and $72 \mathrm{~h}$ for the complexes. Collected raw spectra from all analyses were trans- 
formed by Micromass MassLynx software (version 4.0, Waters Corp.).

\section{Circular Dichroism Spectroscopy}

CD spectra of HsCen-2 at protein concentration $\sim 0.1$ $\mathrm{mM}$ were collected on a J-810 spectropolarimeter (JASCO, Japan) continuously purged by $\mathrm{N}_{2}$ and equipped with a temperature-control system CTC-345. Spectral and temperature-dependent measurements were performed using a U-type quartz cell of path length $0.148 \mathrm{~mm}$ in the far-UV range $(185-250 \mathrm{~nm})$ at a bandwidth of $2 \mathrm{~nm}$, and a rectangular $0.5-\mathrm{cm}$ cell in near-UV range (250-320 nm) at bandwidth of $1 \mathrm{~nm}$ in a custom-built thermostated cell holder. CD spectra were recorded using five accumulations, each at scan speed of $20 \mathrm{~nm} / \mathrm{min}$ and a response time of $2 \mathrm{~s}$. CD spectra were collected at $0{ }^{\circ} \mathrm{C}$. The continuous temperature dependence of the ellipticity at $222 \mathrm{~nm}$ was measured using a scan rate of $60^{\circ} \mathrm{C} / \mathrm{h}$ and a response time of $8 \mathrm{~s}$. Solvent evaporation was prevented by placing a drop of oil (that had been repeatedly boiled in water to remove soluble impurities) on the top of the sample in the cell. $\mathrm{CD}$ spectra in the far-UV range were smoothed by using the JASCO noise reduction routine. CD data are presented in units of molar ellipticity per residue.

\section{Preparation of Samples for CD Spectroscopy}

HsCen-2 or $\Delta 25$ HsCen-2 in $50 \mathrm{mM}$ Tris, $1 \mathrm{mM} \mathrm{Na}$ EDTA, 1 mM DTT, pH 7.4, were dialyzed extensively against $10 \mathrm{mM}$ MOPS, $1 \mathrm{mM}$ EGTA (free acid), $\mathrm{pH} 7.4$ or $60 \mathrm{mM}$ MOPS, $100 \mathrm{mM} \mathrm{KCl}, 1 \mathrm{mM}$ EGTA (with $~ 35$ $\mathrm{mM} \mathrm{Na}^{+}$added from $\mathrm{pH}$ adjustment) $\mathrm{pH}$ to 7.4 (prepared using acid washed glassware) at $4^{\circ} \mathrm{C}$ in a $10 \times 0.5$ $\mathrm{ml}$ microdialyzer, 1000 MWCO SpectraPor 6 dialysis membrane (Spectrum Industries). Dialyzed protein was given a microcentrifuge clean spin at 15,000 $\times g$ for 6 min, and supernatant was placed in a new tube. The protein was then diluted into buffer to give $\sim 0.1 \mathrm{mM}$ protein which was used for CD spectra measurements.

\section{Secondary Structure Calculation}

Secondary structure of HsCen-2 was calculated from $\mathrm{CD}$ spectra in the far-UV spectral range using the CDPro suite of programs [61] - a modified version of three methods: SELCON3 [62], CONTIN/LL-CONTIN method [63] in locally linearized approximation [64], and CDSSTR [65]. In addition to the secondary structure content, programs in the CDPro package also calculate the number and average length of the secondary structure segments. The number of secondary structure segments is calculated by dividing the number of residues included in the distorted helical structure by factor of four and in the distorted $\beta$ structure by factor of two. Dividing the total amount of residues in $\alpha$ helix or $\beta$ strand (ordered plus distorted) by the number of segments gives the average length of the segment.
Table 1. Secondary structure of HsCen-2 in Apo-, $\mathrm{Mg}^{2+}-$, and $\mathrm{Ca}^{2+}$-states at $0^{\circ} \mathrm{C}$ in buffer of high ionic strength $(60 \mathrm{mM}$ MOPS, $100 \mathrm{mM} \mathrm{KCl}, 1 \mathrm{mM}$ EGTA, $\pm 3 \mathrm{mM} \mathrm{MgCl}_{2}$ or $\mathrm{CaCl}_{2}, \mathrm{pH}$ 7.4) calculated from far-UV CD spectra on Figure 9.

\begin{tabular}{|c|c|c|c|}
\hline & Apo-state & $\begin{array}{l}\mathrm{Mg}^{2+}- \\
\text { saturated } \\
\text { state }\end{array}$ & $\begin{array}{c}\mathrm{Ca}^{2+}- \\
\text { saturated } \\
\text { state }\end{array}$ \\
\hline$\alpha_{\text {regular }}$ & $22.7 \pm 1.3$ & $23.4 \pm 1.4$ & $25.2 \pm 2.4$ \\
\hline$\alpha_{\text {distorted }}$ & $17.0 \pm 0.4$ & $17.0 \pm 0.2$ & $17.5 \pm 0.5$ \\
\hline$\alpha_{\text {total }}$ & $39.7 \pm 1.4$ & $40.4 \pm 1.4$ & $42.7 \pm 2.0$ \\
\hline$\beta_{\text {regular }}$ & $4.5 \pm 3.2$ & $5.2 \pm 2.5$ & $5.5 \pm 1.7$ \\
\hline$\beta_{\text {distorted }}$ & $5.9 \pm 0.3$ & $5.8 \pm 0.2$ & $5.3 \pm 0.9$ \\
\hline$\beta_{\text {total }}$ & $10.4 \pm 3.5$ & $11.0 \pm 2.6$ & $10.8 \pm 0.9$ \\
\hline$\beta_{\text {turns }}$ & $19.1 \pm 2.4$ & $18.0 \pm 2.8$ & $16.3 \pm 3.0$ \\
\hline Unordered & $30.7 \pm 2.5$ & $30.8 \pm 1.9$ & $30.0 \pm 0.6$ \\
\hline$N_{\alpha}$ & $7.5 \pm 0.2$ & $7.5 \pm 0.1$ & $7.7 \pm 0.2$ \\
\hline$L_{\alpha}^{a v}$ & $9.4 \pm 0.3$ & $9.5 \pm 0.3$ & $9.8 \pm 0.7$ \\
\hline$N_{\beta}$ & $5.3 \pm 0.4$ & $5.2 \pm 0.2$ & $4.5 \pm 0.7$ \\
\hline$L_{\beta}^{a v}$ & $3.4 \pm 0.9$ & $3.7 \pm 0.7$ & $3.6 \pm 0.8$ \\
\hline
\end{tabular}

$\alpha_{\text {regular }}$ or $\beta_{\text {regular }}$ fraction of residues (\%) in central part of helical segments or $\beta$ strands; $\alpha_{\text {distorted }}$ or $\beta_{\text {distorted }}$ fraction of terminus residues (\%) in helices (two at each end of helix; four total per helical segment) or $\beta$ strands (one residue at each end of strand; two total per strand); $\alpha_{\text {total }}$ or $\beta_{\text {total }}$ total content of $\alpha$ or $\beta$ structure is equal to the sum of the fractions $(\%)$ of regular and distorted residues; $\beta_{\text {turns }}$ fraction of residues (\%) in turns; Unordered, fraction of residues $(\%)$ in unordered structure; $N_{\alpha}$ or $N_{\beta}$, number of helix or $\beta$ strand, $L_{\alpha}^{a v}$ or $L_{\beta}^{a v}$, average number of residues per helix ( $\sim 1.5 \AA$ per residue) or $\beta$ strand ( $\sim 3.4 \AA$ A per residue).

Tertiary structure class was determined by the CLUSTER program [66] of CDPro. Two sets of reference proteins were used for secondary structure calculation: 48 proteins [67], or $23 \alpha+\beta$ reference proteins, which were selected by the CLUSTER program. The average value of the six quantities (three methods and two reference sets) \pm RMS deviations are presented in Table 1 .

\section{Measurement of Protein Concentration}

The spectrophotometric method was used for the concentration determination. Measurements in the near-UV range were done using a DU 640 spectrometer (Beckman) with a spectral bandwidth of $1.8 \mathrm{~nm}$, and employing the apo-centrin $2 \mathrm{M}$ absorptivity of 1.46 $\mathrm{mM}^{-1} \mathrm{~cm}^{-1}$ at $277 \mathrm{~nm}$ obtained by averaging the results from four methods of calculation [68-71]. All spectra were corrected for turbidity by plotting the dependence of the log of the absorbance of the solution versus the $\log$ of the wavelength and extrapolating the linear dependence between these quantities in the range 340$440 \mathrm{~nm}$ to the absorption range $240-300 \mathrm{~nm}$. The extrapolated values of absorbance were then subtracted from the measured value. These corrections decreased the apparent absorbance of protein at $\sim 277 \mathrm{~nm}$ by about $15 \%$. The scatter correction routine of the DU-640 was used for this purpose.

\section{Results and Discussion}

Figure 1 shows the alignment of the four known mammalian centrins. The EF-hand sequences representing 


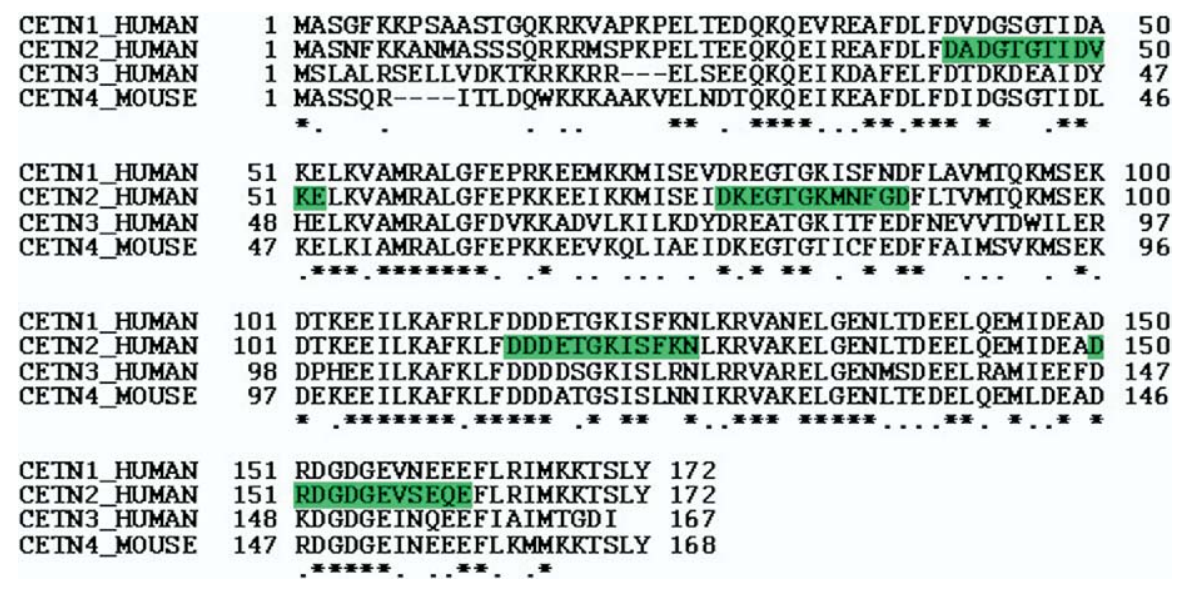

Figure 1. The amino acid sequence of human centrin-1-3 and mouse centrin-4. The four putative EF-hands in human centrin-2 are highlighted in green. Asterisks indicate amino acid identities; period indicate homologous amino acids at that position.

potential calcium/magnesium binding loops are highlighted in green. There is considerable sequence homology among these members of the centrin family. The EF-hand sequences show significant divergence, through substitution of invariant amino acids from canonical EF-hands except for site IV at the C-terminus $[19,72-78]$. Of note is the very basic N-terminus preceding the first EF-hand; this is the unique element in centrin sequences relative to other EF-hand proteins.

\section{Metal Ion Binding Studies}

We showed previously that micro-flow electrospray ionization-mass spectrometry ( $\mu$ ESI-MS) can be used to determine stoichiometries of ion binding to EF-hand and zinc finger proteins and is useful for identifying protein-small ligand, protein-protein and protein-DNA interactions [23-40]. Relative affinities and stoichiometries of metal binding determined by $\mu$ ESI-MS reflect values defined in solution. We, therefore, undertook to identify the metal ion binding stoichiometries for HsCen-2 by $\mu$ ESI-MS.

\section{Apo-HsCen-2 Exists in Two Conformations}

Using $\mu$ ESI-MS in the positive-ion mode and $17 \mu \mathrm{M}$ HsCen-2 in $25 \mathrm{mM}$ ammonium acetate, we determined $\mathrm{Ca}^{2+}$ or $\mathrm{Mg}^{2+}$ binding by preincubation of the protein with metal ions of increasing concentration $(0,50,100$, $200,300,400 \mu \mathrm{M})$. Figure 2 shows the $\mu$ ESI-MS spectrum of apo-HsCen-2 analyzed under optimal conditions for native conformation detection. The inset in Figure 2 shows the $\mu$ ESI-MS spectrum of apo-HsCen-2 analyzed under standard protein denaturing conditions (water, acetonitrile, acid). This spectrum consists of one charge state distribution of charge states $27+$ to $10+$ over the $\mathrm{m} / \mathrm{z}$ range of 700 to 2100 . The calculated mass of HsCen-2, from primary sequence information, is 20148 Da (see Materials and Methods). The $\mu$ ESI-MS mass $\left(\mathrm{M}_{\mathrm{r}}\right)$ under denatured and native conditions indicates
HsCen-2 in the apo-state is of the correct molecular mass.

The presence of two charge state distributions noted in Figure 2 demonstrates two distinct monomer conformations of native HsCen-2. Monomer conformation 1 is represented by a lower $\mathrm{m} / \mathrm{z}$ range charge state distribution $(20+$ to $10+)$ while monomer conformation 2 is represented by the $9+$ and $8+$ charge states. Both conformations share charge states $10+$ and $9+$, however, deconvoluted spectra of the metal ion binding to charge state $10+$ is more reflective of conformation 1 and charge state $9+$ more reflective of conformation 2 . To minimize the influence of shared charge state ions, all metal binding results have been analyzed using the

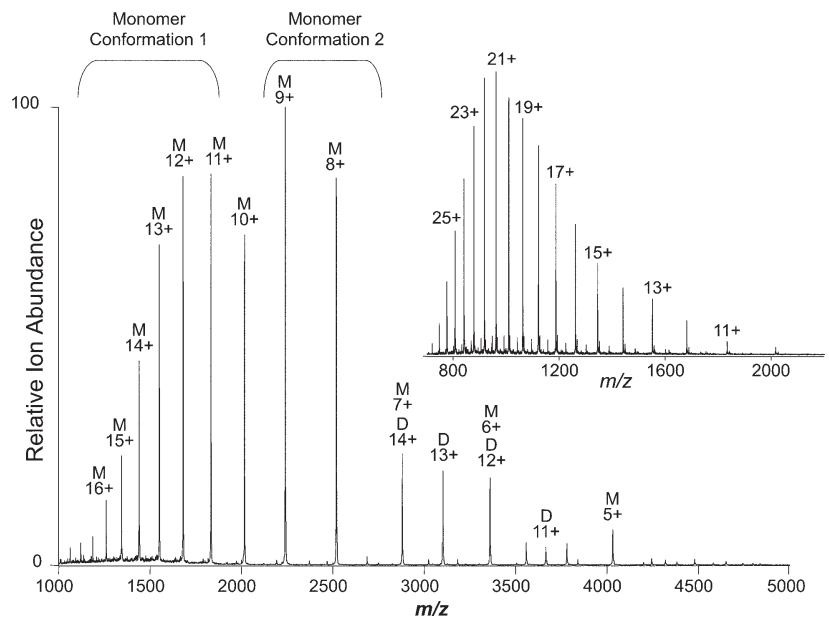

Figure 2. Positive-ion $\mu$ ESI mass spectrum showing the multiply charged ion series for $17 \mu \mathrm{M}$ HsCen-2 in $25 \mathrm{mM}$ ammonium acetate, $\mathrm{pH}$ 7.5. Two different monomeric (M) conformations are attributed to the two charge-state distributions of $\mathrm{m} / \mathrm{z} 1000-2100$ (charge states $20+$ to $10+$ ) and $m / z$ 2100-2700 (charge states +9 and $8+$ ). A dimeric (D) conformation charge state distribution is also observed at $m / z 2600$ to 4100 (charge states $14+$ to $10+$ ). The inset shows the multiply charged ion series for $20 \mu \mathrm{M}$ HsCen-2 under denaturing conditions (water, acetonitrile, acid) with one charge state distribution (charge states $27+$ to $10+$ ). 
$14+$ charge state to represent monomer conformation 1 and the $8+$ charge state to represent monomer conformation 2. Both of these charge states when transformed give an average molecular mass $\left(\mathrm{M}_{\mathrm{r}}\right)$ for HsCen-2 of 20,150 Da (expected value $20148 \mathrm{Da}$ ). Dimeric apoHsCen-2 and a minimal amount of trimeric apoHsCen-2 are also observed in Figure 2. The ions observed at $\mathrm{m} / \mathrm{z} 2880$ and 3360 correspond to the monomer charge states of $7+$ and $6+$ and to the dimer charge states of $14+$ and $12+$ and therefore, were not used in the analysis of the metal binding properties of the monomer.

\section{Apo-HsCen-2 Binds $\mathrm{Ca}^{2+}$ in Both Conformational States}

In Figures 3, 4, 5, and 6 are shown transformed spectra deconvoluted over each of the two charge-state ions, $14+$ and $8+$ representing conformation 1 and conformation 2, respectively. Figure 3 shows data obtained following the titration of $17 \mu \mathrm{M}$ apo-HsCen-2 with increasing amounts of $\mathrm{Ca}^{2+}$. The transformed mass spectra of apo-HsCen-2 are shown in row A; with the addition of $50 \mu \mathrm{M} \mathrm{Ca}^{2+}$ ( $\sim 3: 1 \mathrm{M}$ ratio), row $\mathrm{B}$; and with addition of $100 \mu \mathrm{M} \mathrm{Ca}^{2+}(\sim 6: 1 \mathrm{M}$ ratio), row $\mathrm{C}$. A form of HsCen-2 with one $\mathrm{Ca}^{2+}$ bound to the protein is detected in both conformations, in addition to a substantial amount of apo-HsCen-2. Some HsCen-2 containing two $\mathrm{Ca}^{2+}$ (Figure 3, row C) is detected in conformation 2. A small amount of dimeric apoHsCen-2 and $1 \mathrm{Ca}^{2+}$-dimeric HsCen-2 and $2 \mathrm{Ca}^{2+}$ dimeric HsCen-2 are also detected (data not shown).

With the addition of $200 \mu \mathrm{M} \mathrm{Ca}^{2+}$ ( $\sim 12: 1 \mathrm{M}$ ratio, Figure 6, row A) conformation 1 is converted to more of the HsCen-2 with one $\mathrm{Ca}^{2+}$ relative to that seen at 100 $\mu \mathrm{M}$ added $\mathrm{Ca}^{2+}$, and has detectable $2 \mathrm{Ca}^{2+}-\mathrm{HsCen}-2$. In conformation 2, however, for the first time, the HsCen-2 is mostly in the $\mathrm{Ca}^{2+}$-bound form. In conformation 2, the $1 \mathrm{Ca}^{2+}-\mathrm{HsCen}-2$ is detected as the most abundant species with a significant amount of $2 \mathrm{Ca}^{2+}-\mathrm{HsCen}-2$, and detectable $3 \mathrm{Ca}^{2+}-\mathrm{HsCen}-2$. On increasing the amount of added $\mathrm{Ca}^{2+}$ to $300 \mu \mathrm{M}(\sim 18: 1 \mathrm{M}$ ratio, Figure 3 , row D), conformation 1 has more of both the HsCen-2 with one and two $\mathrm{Ca}^{2+}$ and detectable $3 \mathrm{Ca}^{2+}-\mathrm{HsCen}-2$.

Conformation 2, (Figure 3, row D), however, clearly has the majority of the protein in the $\mathrm{Ca}^{2+}$-bound form. Most HsCen-2 in conformation 2 appears to have either one (most abundant) or two (next most abundant) $\mathrm{Ca}^{2+}$ bound. A significant amount of $3 \mathrm{Ca}^{2+}-\mathrm{HsCen}-2$ is present, and a $4 \mathrm{Ca}^{2+}$ form of HsCen-2 is observed. Detectable amounts of $5 \mathrm{Ca}^{2+}-\mathrm{HsCen}-2$ and $6 \mathrm{Ca}^{2+}$ HsCen-2 observed may be due to the addition of large amounts of $\mathrm{Ca}^{2+}$. On increasing the $\mathrm{Ca}^{2+}$ addition to $400 \mu \mathrm{M} \mathrm{Ca}^{2+}$ ( 24:1 $\mathrm{M}$ ratio, data not shown) the detectable amounts of the 1, 2 and $3-\mathrm{Ca}^{2+}$-forms of conformation 1 HsCen-2 have modestly increased, and a $4 \mathrm{Ca}^{2+}$ form of HsCen-2 is detectable, although it is a minor amount of the total. Conformation 2, by contrast,
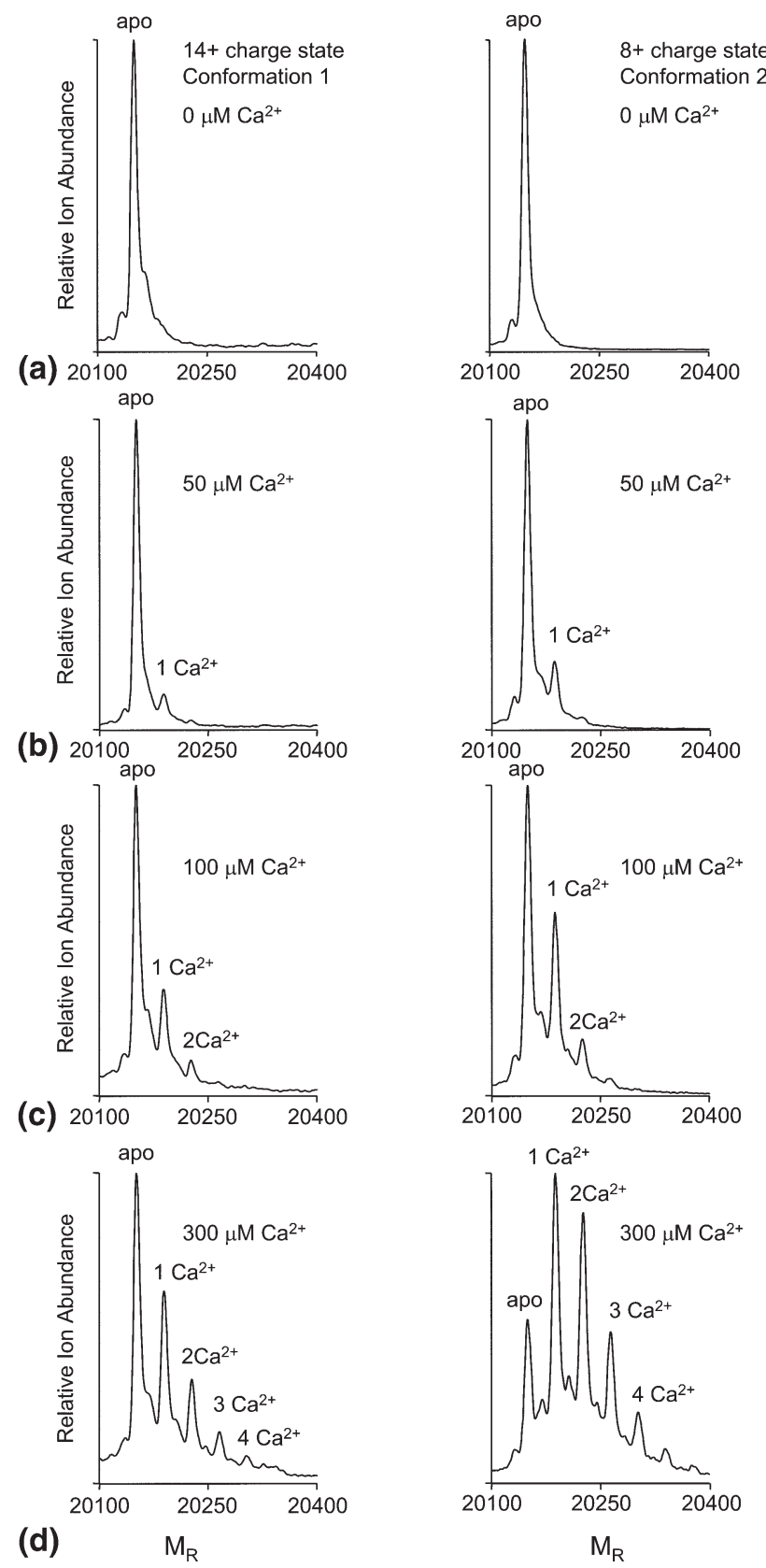

Figure 3. Positive-ion $\mu \mathrm{ESI}$ transformed mass spectra of $17 \mu \mathrm{M}$ HsCen-2 from the 14+ charge state (Monomeric Conformation 1, Panel 1) and the $8+$ charge state (Monomeric Conformation 2, Panel 2) for a calcium metal ion titration; (a) apo-HsCen-2 with no added metal ions. (b) HsCen-2 plus $50 \mu \mathrm{M}$ calcium. (c) HsCen-2 plus $100 \mu \mathrm{M}$ calcium. (d) HsCen-2 plus $300 \mu \mathrm{M}$ calcium.

now exists as $2 \mathrm{Ca}^{2+}$-HsCen-2 as the most abundant single species, with $1 \mathrm{Ca}^{2+}$-HsCen-2 only slightly less abundant. Significant amounts of $3 \mathrm{Ca}^{2+}-\mathrm{HsCen-2}$ are present for conformation 2, with $4 \mathrm{Ca}^{2+}-\mathrm{HsCen}-2$ easily detected. Thus, HsCen-2 in conformation 2, loads with $\mathrm{Ca}^{2+}$ more rapidly, and more completely, than HsCen-2 in conformation 1. The charge-state distribution of conformations 1 and 2 in the $\mu$ ESI-MS untransformed spectra (see Figure 2) were similar in the presence of $0-400 \mu \mathrm{M} \mathrm{Ca}^{2+}$ (data not shown). 

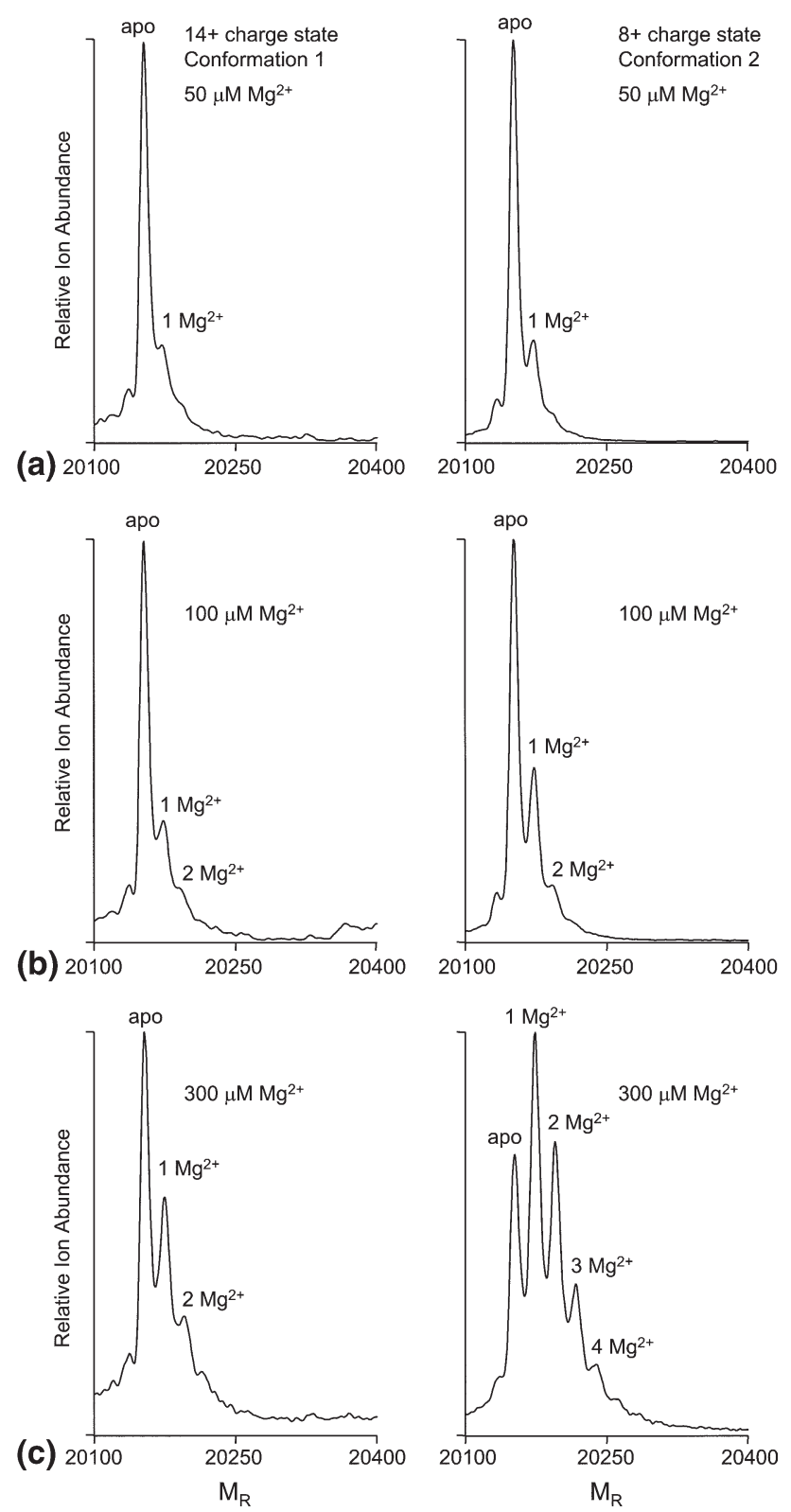

Figure 4. Positive-ion $\mu \mathrm{ESI}$ transformed mass spectra of $17 \mu \mathrm{M}$ HsCen-2 from the 14+ charge state (Monomeric Conformation 1, Panel 1) and the $8+$ charge state (Monomeric Conformation 2, Panel 2) for a magnesium metal ion titration; (a), HsCen-2 plus 50 $\mu \mathrm{M}$ magnesium. (b) HsCen-2 plus $100 \mu \mathrm{M}$ magnesium. (c) HsCen-2 plus $300 \mu \mathrm{M}$ magnesium.

\section{Apo-HsCen-2 Binds $\mathrm{Mg}^{2+}$ in Both Conformational States}

We similarly undertook titration of HsCen-2 $(17 \mu \mathrm{M})$ by incubation of protein with $\mathrm{Mg}^{2+}$ of increasing concentration $(0,50,100,200,300,400 \mu \mathrm{M})$ to determine the $\mathrm{Mg}^{2+}$ binding. Figure 4 shows titration of $17 \mu \mathrm{M}$ apo-HsCen-2 with increasing amounts of $\mathrm{Mg}^{2+}$. In the transformed spectra of HsCen-2 with the addition of $50 \mu \mathrm{M} \mathrm{Mg}^{2+}$ $(\sim 3: 1 \mathrm{M}$ ratio, row $\mathrm{A})$ and $100 \mu \mathrm{M} \mathrm{Mg}^{2+}(\sim 6: 1 \mathrm{M}$ ratio, row B), a form of HsCen-2 containing one $\mathrm{Mg}^{2+}$ is detected for both conformations, although it appears more prominent for conformation 2. With $100 \mu \mathrm{M} \mathrm{Mg}^{2+}$ most of the HsCen-2 remains in the apo-form. Some $2 \mathrm{Mg}^{2+}$ HsCen-2 (Figure 4, row B) is detected for conformation 2. A small amount of dimeric apo-HsCen-2 and dimeric HsCen-2 with one and two $\mathrm{Mg}^{2+}$ are also detected (data not shown). Adding $200 \mu \mathrm{M} \mathrm{Mg}^{2+}(\sim 12: 1 \mathrm{M}$ ratio, Figure 5 , row A) resulted in increased amounts of the $1 \mathrm{Mg}^{2+}$ HsCen-2 and some $2 \mathrm{Mg}^{2+}-\mathrm{HsCen}-2$ in conformation 2. With the addition of $300 \mu \mathrm{M} \mathrm{Mg}^{2+}(\sim 18: 1 \mathrm{M}$ ratio, Figure 4 , row $\mathrm{C})$, conformation 1 of the protein shows increased $1 \mathrm{Mg}^{2+}$-HsCen-2 and some $2 \mathrm{Mg}^{2+}-\mathrm{HsCen}-2$ (Figure 4, row $\mathrm{C}$ ). The amounts of 1 and $2 \mathrm{Mg}^{2+}$-HsCen-2 are less than the apo-HsCen-2 in conformation 1. Conformation 2,
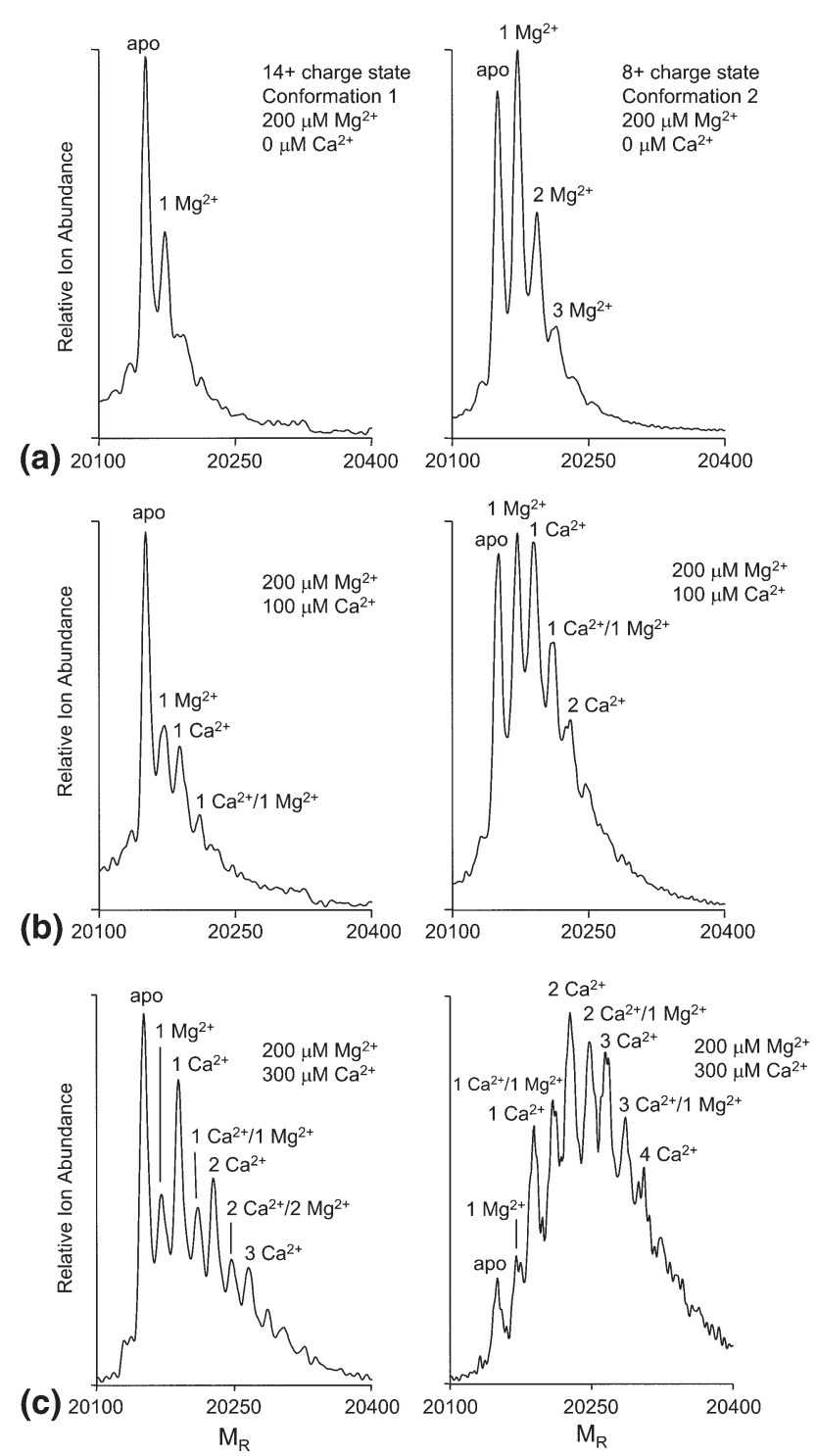

Figure 5. Competition between calcium and magnesium ion uptake. Positive-ion $\mu$ ESI transformed mass spectra of HsCen-2 from the $14+$ charge state (Monomeric Conformation 1, Panel 1) and the $8+$ charge state (Monomeric Conformation 2, Panel 2) for a calcium titration into $17 \mu \mathrm{M}$ HsCen-2 preincubated with $200 \mu \mathrm{M}$ magnesium; (a) HsCen-2 with magnesium. (b) HsCen-2 with magnesium plus $100 \mu \mathrm{M}$ calcium. (c) HsCen-2 with magnesium plus $300 \mu \mathrm{M}$ calcium. 

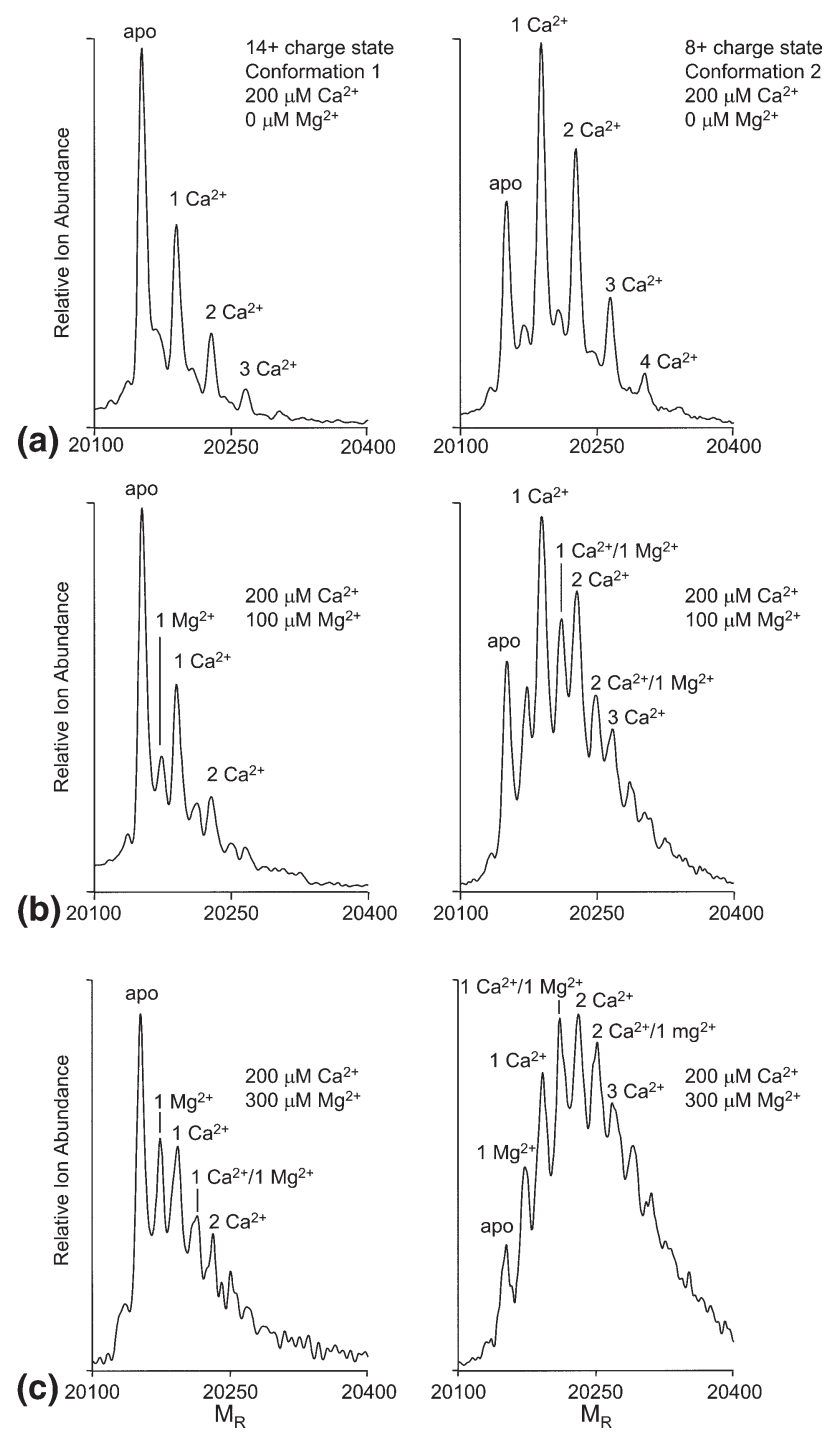

Figure 6. Competition between calcium and magnesium ion uptake. Positive-ion $\mu$ ESI transformed mass spectra of HsCen-2 from the 14+ charge state (Monomeric Conformation 1, Panel 1) and the $8+$ charge state (Monomeric Conformation 2, Panel 2) for a magnesium titration into $17 \mu \mathrm{M}$ HsCen-2 preincubated with 200 $\mu \mathrm{M}$ calcium; (a) HsCen-2 with calcium. b) HsCen-2 with calcium plus $100 \mu \mathrm{M}$ magnesium. (c) HsCen-2 with calcium plus $300 \mu \mathrm{M}$ magnesium

by contrast, has most of the HsCen-2 with one or more $\mathrm{Mg}^{2+}$ bound. The $1 \mathrm{Mg}^{2+}-\mathrm{HsCen}-2$ is the most abundant species, followed by apo-HsCen-2, $2 \mathrm{Mg}^{2+}-\mathrm{HsCen}-2$ and some $3 \mathrm{Mg}^{2+}$-HsCen-2. Addition of $400 \mu \mathrm{M} \mathrm{Mg}^{2+}(\sim 24: 1$ $\mathrm{M}$ ratio, data not shown) resulted in some addition of $\mathrm{Mg}^{2+}$, seen as the 1- and $2 \mathrm{Mg}^{2+}-\mathrm{HsCen}-2$ forms of conformation 1. For conformation 2, the 1- and $2 \mathrm{Mg}^{2+}$ HsCen-2 are the most abundant species followed by apo-HsCen-2 and $3 \mathrm{Mg}^{2+}-\mathrm{HsCen}-2$. Some $4 \mathrm{Mg}^{2+}-\mathrm{HsCen}-2$ is detected. Thus, as with the $\mathrm{Ca}^{2+}$ titrations, conformation 2 loads more readily with $\mathrm{Mg}^{2+}$ and to a greater extent than does conformation 1. In fact, up to 4 (and maybe 5 and 6) $\mathrm{Mg}^{2+}$ are taken up at $400 \mu \mathrm{M} \mathrm{Mg}^{2+}$, but one $\mathrm{Mg}^{2+}$ binding site has, by far, the highest affinity, with a second site of significantly lower affinity. The third and fourth $\mathrm{Mg}^{2+}$ bound to HsCen-2 represent the lowest affinity binding sites for $\mathrm{Mg}^{2+}$ on the protein.

\section{Competition Experiments}

Competition experiments were then carried out by first titrating increasing amounts of $\mathrm{Ca}^{2+}$ into HsCen-2 preincubated with $200 \mu \mathrm{M} \mathrm{Mg}^{2+}$ followed by experiments in which increasing amounts of $\mathrm{Mg}^{2+}$ were titrated into HsCen-2 preincubated with $200 \mu \mathrm{M} \mathrm{Ca}^{2+}$. The first metal was incubated with protein for $30 \mathrm{~min}$, and then for an additional $30 \mathrm{~min}$ in the presence of the competing metal before being analyzed by $\mu$ ESI-MS.

We first added increasing amounts of $\mathrm{Ca}^{2+}$ to HsCen-2 preincubated with $\mathrm{Mg}^{2+}$. Without $\mathrm{Ca}^{2+}$ addition in the presence of the $200 \mu \mathrm{M} \mathrm{Mg}^{2+}$, some $1 \mathrm{Mg}^{2+}$-HsCen-2 is detected for conformation 1. (Figure 5, row A). Conformation 2 (Figure 5, row A) exists with as much $1 \mathrm{Mg}^{2+}$ HsCen-2 as it does as apo-HsCen-2, a significant amount of $2 \mathrm{Mg}^{2+}-\mathrm{HsCen}-2$, and detectable amounts of $3 \mathrm{Mg}^{2+}$ HsCen-2. With $50 \mu \mathrm{M}$ added $\mathrm{Ca}^{2+}$ there is the obvious observation of a peak representing $1 \mathrm{Ca}^{2+-} \mathrm{HsCen}-2$ for the protein in conformation 1 (data not shown), without diminution in the abundance of the $1 \mathrm{Mg}^{2+}-\mathrm{HsC}$ - 2 . Similarly, for conformation 2 (data not shown), $50 \mu \mathrm{M}$ $\mathrm{Ca}^{2+}$ gives a significant amount of $1 \mathrm{Ca}^{2+}-\mathrm{HsCen}-2$ without diminution of the $1 \mathrm{Mg}^{2+}-\mathrm{HsCen}-2$. A peak also appears representing a $1 \mathrm{Mg}^{2+} / 1 \mathrm{Ca}^{2+}-\mathrm{HsCen}-2$ species. Calcium seems to be loading at a relatively $\mathrm{Ca}^{2+}$-specific site, a site different from that binding one $\mathrm{Mg}^{2+}$. This is also indicated by the observation of a peak representing $1 \mathrm{Mg}^{2+} / 1 \mathrm{Ca}^{2+}-\mathrm{HsCen}-2$. On incubation with $100 \mu \mathrm{M}$ $\mathrm{Ca}^{2+}$, conformation 1 (Figure 5, row B) exists with somewhat less $1 \mathrm{Mg}^{2+}$-HsCen-2 but the abundance of $1 \mathrm{Ca}^{2+}$ HsCen-2 remains similar, and the formation of some of the $1 \mathrm{Ca}^{2+} / 1 \mathrm{Mg}^{2+}-\mathrm{HsCen}-2$ is seemingly at the expense of the $1 \mathrm{Ca}^{2+}$-HsCen-2 form.

For conformation 2 (Figure 5, row B), addition of 100 $\mu \mathrm{M} \mathrm{Ca}^{2+}$ produces somewhat less $1 \mathrm{Mg}^{2+}-\mathrm{HsCen}-2$ and more $1 \mathrm{Ca}^{2+}-\mathrm{HsCen}-2$ and the mixed $1 \mathrm{Mg}^{2+} / 1 \mathrm{Ca}^{2+}$ HsCen-2. On incubation with $200 \mu \mathrm{M} \mathrm{Ca}^{2+}$ (data not shown), conformation 1 exists as comparable amounts of $1 \mathrm{Mg}^{2+}-\mathrm{HsCen}-2$ as at $100 \mu \mathrm{M} \mathrm{Ca}^{2+}$, but more $1 \mathrm{Ca}^{2+}$ HsCen-2 and $1 \mathrm{Mg}^{2+} / 1 \mathrm{Ca}^{2+}-\mathrm{HsCen}-2$ forms. In addition, some $2 \mathrm{Ca}^{2+}-\mathrm{HsCen}-2$ and $2 \mathrm{Ca}^{2+} / 1 \mathrm{Mg}^{2+}-\mathrm{HsCen}-2$ are present. Most of the apo- and $1 \mathrm{Mg}^{2+}-\mathrm{HsCen}-2$ of conformation 2 are lost, and possibly the $1 \mathrm{Ca}^{2+}-\mathrm{HsCen}-2$ diminishes in favor of increasing amounts of $2 \mathrm{Ca}^{2+}-\mathrm{HsCen}-2$ and $1 \mathrm{Mg}^{2+} / 1 \mathrm{Ca}^{2+}-\mathrm{HsCen}-2$; the $2 \mathrm{Ca}^{2+}-\mathrm{HsCen}-2$ becomes the most abundant species along with $2 \mathrm{Ca}^{2+} / 1 \mathrm{Mg}^{2+}$ HsCen-2 and significant amounts of $3 \mathrm{Ca}^{2+}-\mathrm{HsCen}-2$. For the titration with $300 \mu \mathrm{M} \mathrm{Ca}^{2+}$, conformation 1 (Figure 5, row C) changes little from the $200 \mu \mathrm{M} \mathrm{Ca}^{2+}$ incubation but for a slight increase in $2 \mathrm{Ca}^{2+}-\mathrm{HsCen}-2$ and possibly $2 \mathrm{Ca}^{2+} / 1 \mathrm{Mg}^{2+}-\mathrm{HsCen}-2$ and the appearance of detectable amounts of $3 \mathrm{Ca}^{2+}-\mathrm{HsCen}-2$. Conformation 2 (Figure 5, row C) also changes little from the $200 \mu \mathrm{M} \mathrm{Ca}^{2+}$ incubation but for the loss of more $1 \mathrm{Mg}^{2+}-\mathrm{HsCen}-2$ and $1 \mathrm{Mg}^{2+} /$ 
$1 \mathrm{Ca}^{2+}-\mathrm{HsCen}-2$ owing to increasing amounts of $3 \mathrm{Ca}^{2+}-$ HsCen-2 and emerging amounts of $3 \mathrm{Ca}^{2+} / 1 \mathrm{Mg}^{2+}$ HsCen-2 and $4 \mathrm{Ca}^{2+}$-HsCen-2.

We next added increasing amounts of $\mathrm{Mg}^{2+}$ to $\mathrm{HsCen}-2$ preincubated with $\mathrm{Ca}^{2+}$. Without $\mathrm{Mg}^{2+}$ addition in the presence of the $200 \mu \mathrm{M} \mathrm{Ca}^{2+}$, some $1 \mathrm{Ca}^{2+}$ - and $2 \mathrm{Ca}^{2+}-\mathrm{HsCen}-2$ in conformation 1 are produced (Figure 6, row A). Conformation 2 (Figure 6, row A) overall takes up more $\mathrm{Ca}^{2+}$ than does conformation 1 , and exists more as $1 \mathrm{Ca}^{2+}-\mathrm{HsCen}-2$ as well as apo-HsCen-2 and a significant amount of $2 \mathrm{Ca}^{2+}$-HsCen-2 including detectable amounts of $3 \mathrm{Ca}^{2+}$ and $4 \mathrm{Ca}^{2+}-\mathrm{HsCen}-2$. With addition of $50 \mu \mathrm{M}$ $\mathrm{Mg}^{2+}$, conformation 1 (data not shown) is little changed and $\mathrm{Mg}^{2+}$ binding is unclear. Conformation 2 (data not shown), however, clearly exists as small amounts of $1 \mathrm{Mg}^{2+}$ HsCen-2 and $1 \mathrm{Mg}^{2+} / 1 \mathrm{Ca}^{2+}-\mathrm{HsCen}-2$, and the complexes seen before $\mathrm{Mg}^{2+}$ addition are little changed. Addition of $100 \mu \mathrm{M} \mathrm{Mg}^{2+}$ leaves conformation 1 (Figure 6 , row B) only slightly changed, whereas for conformation 2 (Figure 6, row B), significant amounts of $1 \mathrm{Mg}^{2+}$ HsCen-2, $1 \mathrm{Ca}^{2+} / 1 \mathrm{Mg}^{2+}$-HsCen-2 and $2 \mathrm{Ca}^{2+} / 1 \mathrm{Mg}^{2+}$ HsCen-2 are now detected.

After addition of $200 \mu \mathrm{M} \mathrm{Mg}^{2+}$, conformation 1 (data not shown) exists with the amounts of $1 \mathrm{Ca}^{2+}-\mathrm{HsCen}-2$ and $2 \mathrm{Ca}^{2+}-\mathrm{HsCen}-2$ relatively unchanged, but $1 \mathrm{Mg}^{2+}$ HsCen-2 and $1 \mathrm{Ca}^{2+} / 1 \mathrm{Mg}^{2+}-\mathrm{HsCen}-2$ now appear. Conformation 2 with $200 \mu \mathrm{M} \mathrm{Mg}^{2+}$, (data not shown), in contrast to conformation 1, has little apo-protein remaining, and the amount of $1 \mathrm{Mg}^{2+}-\mathrm{HsCen}-2$ is increased. $1 \mathrm{Ca}^{2+} / 1 \mathrm{Mg}^{2+}-\mathrm{HsCen}-2$ and $2 \mathrm{Ca}^{2+} / 1 \mathrm{Mg}^{2+}$-HsCen-2 now increase while the peak height for $1 \mathrm{Ca}^{2+}-\mathrm{HsCen}-2$ decreases, suggesting it is converted to a complex also containing one $\mathrm{Mg}^{2+}$ or one $\mathrm{Mg}^{2+}$ and one $\mathrm{Ca}^{2+}$. Addition of $300 \mu \mathrm{M} \mathrm{Mg}^{2+}$ slightly increased the amounts of $1 \mathrm{Mg}^{2+}$ HsCen-2 and $1 \mathrm{Mg}^{2+} / 1 \mathrm{Ca}^{2+}-\mathrm{HsCen}-2$ in conformation 1 (Figure 6, row C). The pattern of metal binding in conformation 2 in the presence of $300 \mu \mathrm{M} \mathrm{Mg}^{2+}$ is similar to that observed to that with $200 \mu \mathrm{M} \mathrm{Mg}^{2+}$. Overall, $\mathrm{Ca}^{2+}$ more readily displaced $\mathrm{Mg}^{2+}$, than $\mathrm{Mg}^{2+}$ displaced $\mathrm{Ca}^{2+}$, indicating a greater affinity for $\mathrm{Ca}^{2+}$ at those metal-binding sites.

\section{Binding of XPC Peptide to HsCen-2 and the Effect of $\mathrm{Ca}^{2+}$ on Complex Formation}

Figure 7 shows the $\mu$ ESI-MS spectra of $52 \mu \mathrm{M}$ apoHsCen-2. In row $\mathrm{A}$, monomeric $(\mathrm{M})$ and dimeric protein (D) are seen. In row B, adding XPC peptide to the apo-HsCen-2 ( $\sim 6: 1$, peptide:HsCen-2) is seen to cause the disappearance of dimeric HsCen-2 (D) and also a decrease of charge states $9+$ and $8+$ compared to charge states $11+$ and $10+$. The spectra shows the appearance of the complex of 1 XPC peptide with one molecule of HsCen-2 (M + XPC), or two molecules of XPC peptide with one molecule of HsCen-2 $(\mathrm{M}+2 \mathrm{XPC})$. The decrease in the amount of the $9+$ and $8+$ charge states suggests the binding of the peptide to monomer conformation 2 . The disappearance of the dimer may be due to instability of the complex as a

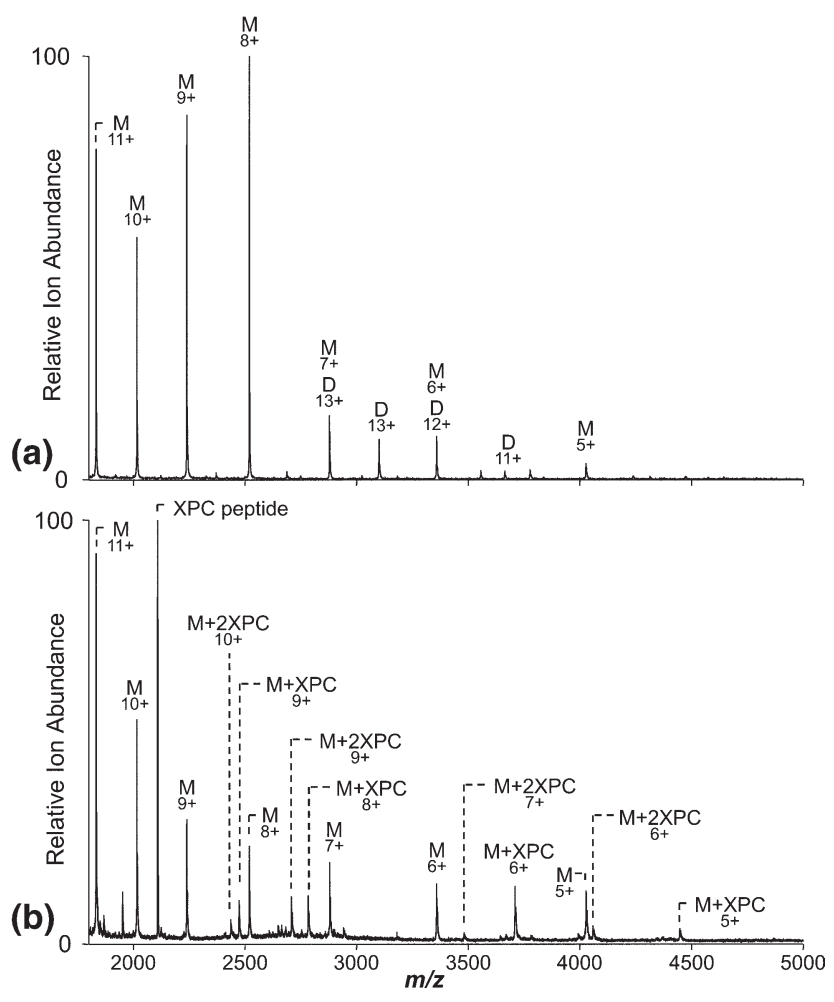

Figure 7. Positive ion $\mu \mathrm{ESI}$ mass spectra of the multiply charged ion series of $52 \mu \mathrm{M}$ HsCen-2 incubated with XPC peptide for 30 min. (a) apo HsCen-2 with no added peptide or metal ions. (M, monomer; D, dimer) (b) HsCen-2 plus $290 \mu \mathrm{M}$ XPC peptide. (M, monomer; $\mathrm{M}+\mathrm{XPC}$, monomer plus XPC peptide)

result of the large excess of peptide ions present. With addition of $\mathrm{Ca}^{2+}$ the profile is similar to that in Figure 7, row $\mathrm{B}$, with the $\mathrm{Ca}^{2+}$-bound forms of HsCen-2 and both single and double-molecule XPC bound to HsCen-2-Ca ${ }^{2+}$ (data not shown); no dimeric HsCen-2 is observable. The results change little for incubations extended up to $72 \mathrm{~h}$ (data not shown).

\section{Metal-Induced Change in HsCen-2 Conformation Assessed by Mass Spectrometry}

We previously demonstrated that monitoring gross protein secondary and tertiary structural changes upon metal ion binding can be accomplished by $\mu$ ESI-MS. Changes in charge state distributions upon $\mathrm{Ca}^{2+}$ binding by calbindin $\mathrm{D}_{28 \mathrm{~K}}$ and DREAM/calsenilin, and zinc binding by the vitamin D receptor DNA binding domain, correlated with changes in CD and fluorescence spectra $(24,25,27-29,32)$. Calcium and magnesium addition to apo-HsCen-2 did not change the charge-state distributions upon metal binding, indicating no gross change in protein conformation.

We next examined $\Delta 25 \mathrm{HsCen}-2$ centrin because the $\mathrm{NH}_{2}$ terminus is believed to play role in centrin selfassembly when high concentrations of $\mathrm{Ca}^{2+}$ and centrin are present. By deleting the first 25 amino acids self assembly is abolished. Using $\mu$ ESI-MS in the positive mode and $17 \mu \mathrm{M} \Delta 25 \mathrm{HsCen}-2$ in $25 \mathrm{mM}$ ammonium acetate, we determined the $\mathrm{Ca}^{2+}$ and $\mathrm{Mg}^{2+}$ binding by 


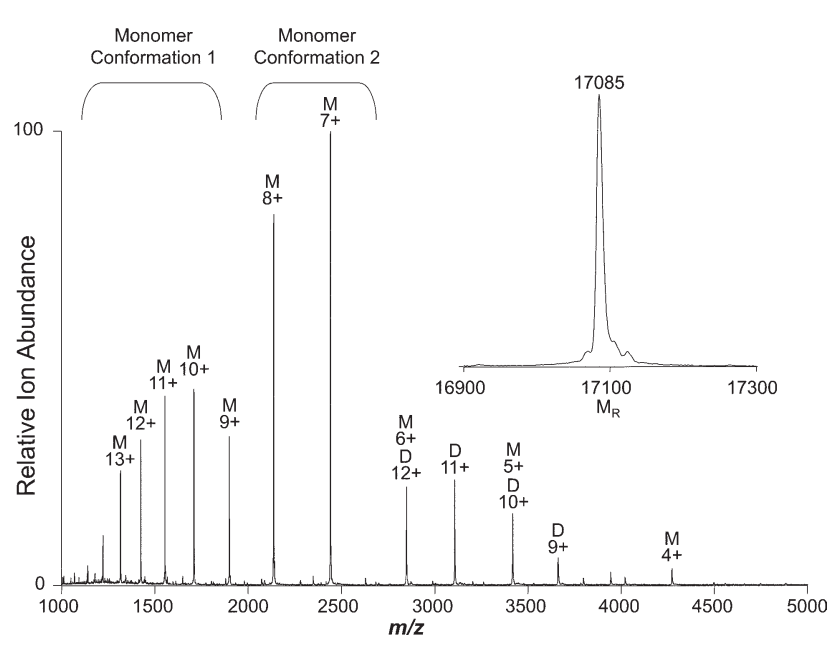

Figure 8. Positive ion $\mu$ ESI mass spectra of the multiply charged ion series of $17 \mu \mathrm{M} \Delta 25 \mathrm{HsCen}-2$ in $25 \mathrm{mM}$ ammonium acetate, $\mathrm{pH}$ 7.5. Two different monomeric conformations are observed at $\mathrm{m} / \mathrm{z}$ 1000-2000 (charge states $14+$ to $9+$ ) and $m / z$ 2000-2500 (charge states $8+$ and $7+$ ). The inset shows the transformed mass spectrum of the apo $\Delta 25 \mathrm{HsCen}-2$.

preincubation of protein with metal ions of increasing concentration $(0,50,100,200,300,400 \mu \mathrm{M})$. The calculated mass of this $\Delta 25 \mathrm{HsCen}-2$ construct, from primary sequence, is $17084 \mathrm{Da}$ (see Materials and Methods). The $\mu$ ESI-MS mass $\left(\mathrm{M}_{\mathrm{r}}\right)$ indicates all the $\Delta 25 \mathrm{HsCen}-2$ is in the apo-state and of the correct mass (Figure 8). In general, the metal-binding characteristics of $\Delta 25 \mathrm{HsCen}-2$ are similar to those of the full-length protein (data not shown).

\section{Spectroscopy}

Far- and near-UV CD spectra of HsCen-2 are shown in Figure 9. The shape and amplitude of far-UV CD spectra are characteristics of proteins with a high content of helical structure. Only small changes in the far-UV spectra of HsCen-2, as well as $\Delta 25 \mathrm{HsCen-2} \mathrm{(data} \mathrm{not} \mathrm{shown)} \mathrm{occur}$ in the presence of added calcium or magnesium. For example, the molar ellipticity at $222 \mathrm{~nm}$ increases $6.1 \%$ in the $\mathrm{Mg}^{2+}$-saturated and $11.8 \%$ in the $\mathrm{Ca}^{2+}$-saturated states in comparison to the apo-state. The isobestic point of all three curves in Figure 9 is close to $203 \mathrm{~nm}$. All these changes in the far-UV CD spectra are consistent with an increase in helical content of the protein upon bivalent cation binding.

The near-UV CD spectra (Figure 9, insert) do not show any signal from the only tyrosine residue (Tyr 172) in the protein and instead show three negative signals at $\sim 255$, $\sim 262$, and $\sim 268 \mathrm{~nm}$ belonging to the 10 phenylalanines (Phe) of HsCen-2. Differential near-UV CD spectra between cation-bound states and the apo-state (data not shown) demonstrate the absence of changes in tertiary structure upon $\mathrm{Mg}^{2+}$-binding (apparent changes are due to changes in amplitude of the far-UV CD background in near-UV range). An analysis of the spectral changes with $\mathrm{Ca}^{2+}$-binding shows that an increase in asymmetry of microenvironments of Phe occurs $(\sim 28 \%$ change over background), and this is indicative of changes in protein tertiary structure.

Far- and near-UV CD spectra of the protein at $0{ }^{\circ} \mathrm{C}$ in buffer of high or low ionic strength are similar (data not shown). Table 1 shows results from the calculation of secondary structure of HsCen-2 from the far-UV CD spectra in Figure 1. Even though there are small increases in the amplitude of the CD spectra, the secondary structure of HsCen-2 in the apo- or cation-bound states is practically the same. The only significant difference (larger than the RMS deviation) is an increase of $3 \pm 2 \%(\sim 5$ residues) of the helical structure content upon $\mathrm{Ca}^{2+}$ binding. Protein helical content is $\sim 43 \%$ ( $\sim 73$ residues) in the $\mathrm{Ca}^{2+}$-saturated and $\sim 40 \%$ in the apo-state ( $\sim 68$ residues). $\beta$-sheet content is $\sim 11 \%$ (18-19 residues), $\beta$-turns are $\sim 18 \%$ (28-32 residues), and unordered structure is $\sim 31 \%$ (52-53 residues) for all states of the protein. Residues included in helical structure form 7-8 segments with average length of $9-10$ residues (13-15 $\AA$ ), and in the $\beta$-sheets, $4-5$ strands have average lengths of $3-4$ residues (10-14 $⿱$ A).

Cluster analysis of far-UV CD spectra [55] shows that HsCen-2 belongs to the $\alpha+\beta$ tertiary structure class proteins. Earlier reports presented similar conclusions from CD measurements on HsCen-2 following $\mathrm{Ca}^{2+}$ addition $[19,79,80]$. Durussel et al. showed that the protein had a higher helix content ( $57 \% \alpha$-helix $+3_{10}$ helix versus $43 \%$, Table 1 ) and lower $\beta$-sheet content ( $3 \%$ versus $\sim 11 \%$, Table 1). They found a similar percentage of $\beta$-turns $(15 \%$ turns versus $\sim 18 \%$, Table 1 ). The differences in amplitude [80] and shape of the CD spectra and/or secondary structure content [19] can be attributed to differences in the protein concentration and/or differences in the methods by which secondary structure was calculated.

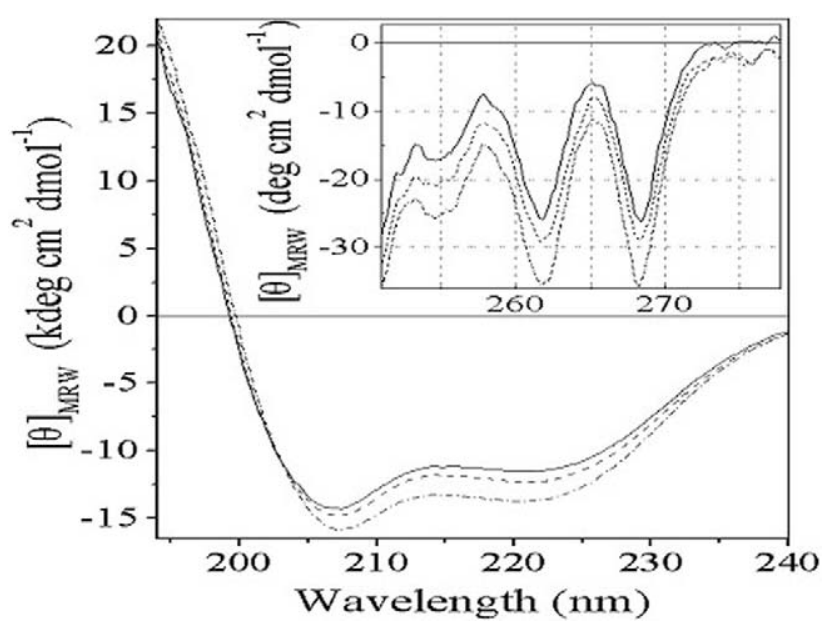

Figure 9. Far- and near-UV (insert) CD spectra of HsCen-2 in the apo state (60 mM MOPS, $100 \mathrm{mM} \mathrm{KCl,} 1 \mathrm{mM}$ EGTA, pH 7.4), in the $\mathrm{Mg}^{2+}$-saturated state $(60 \mathrm{mM}$ MOPS, $100 \mathrm{mM} \mathrm{KCl}, 1 \mathrm{mM}$ EGTA, $3 \mathrm{mM} \mathrm{MgCl}_{2}, \mathrm{pH} \mathrm{7.4)}$, and in the $\mathrm{Ca}^{2+}$ saturated state $(60 \mathrm{mM}$

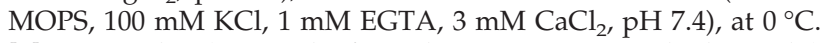
$[\theta]_{\mathrm{MRW}}$, molar (per mol of residues or mean residual weight) ellipticity; Apo-state (solid line), with addition of $3 \mathrm{mM} \mathrm{Mg}^{2+}$ (dashed line), with addition of $3 \mathrm{mM} \mathrm{Ca}^{2+}$ (dash-dot-dot-dot line). 

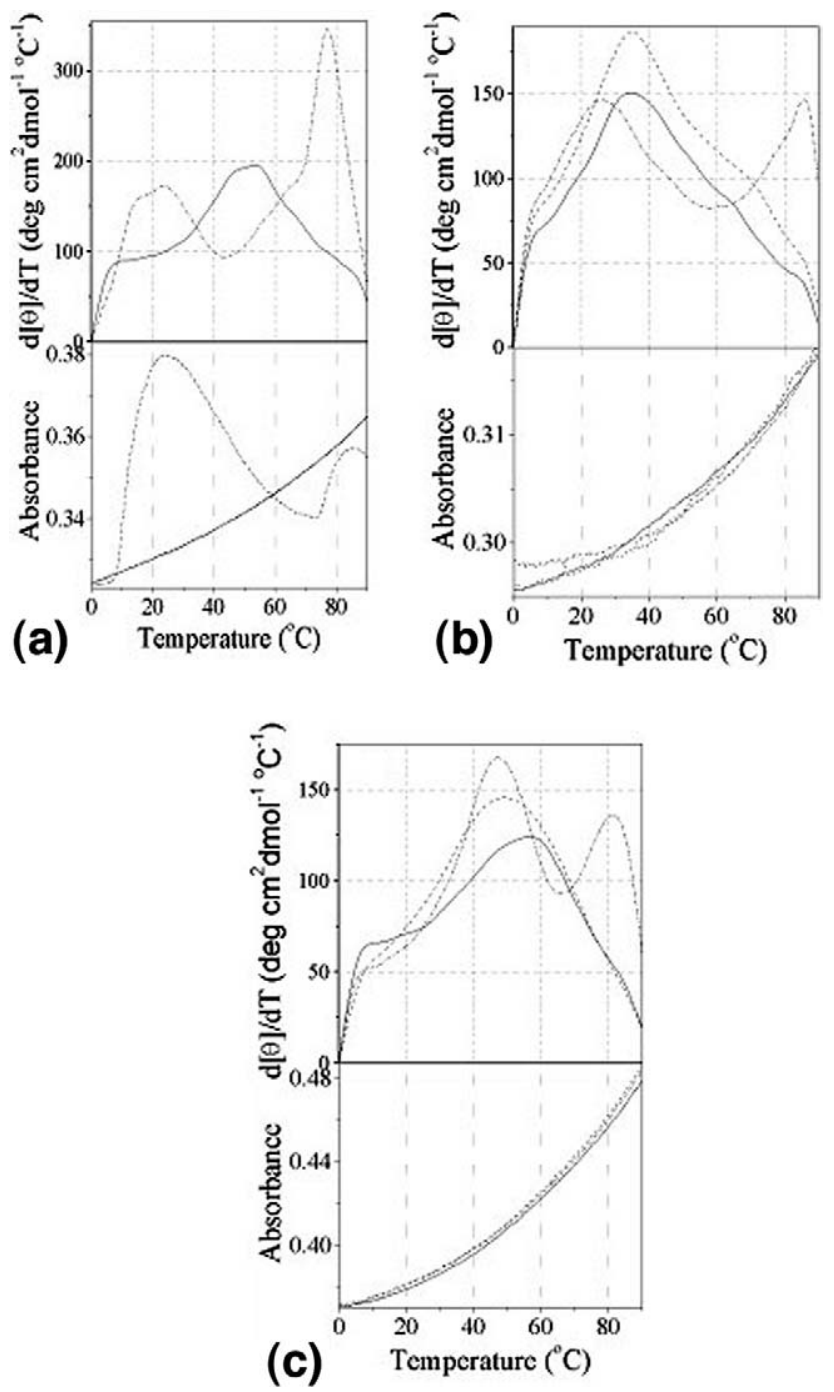

Figure 10. Temperature dependence of the first derivative of the CD signal at $222 \mathrm{~nm}$ and absorbance at $222 \mathrm{~nm}$ of HsCen-2. (a) Protein in buffer of low ionic strength ( $10 \mathrm{mM}$ MOPS, $1 \mathrm{mM}$ EGTA, $\pm 3 \mathrm{mM} \mathrm{CaCl}_{2}, \mathrm{pH} 7.4$ ). (b) $\Delta 25 \mathrm{HsCen}-2$ truncated protein (26-172 HsCen-2) in buffer of low ionic strength. (c) Protein in buffer of high ionic strength (60 mM MOPS, $100 \mathrm{mM} \mathrm{KCl}, 1 \mathrm{mM}$ EGTA, $\pm 3 \mathrm{mM} \mathrm{MgCl} 2$ or $\mathrm{CaCl}_{2}, \mathrm{pH} 7.4$ ); Apo-state (solid line), with addition of $3 \mathrm{mM} \mathrm{Mg}^{2+}$ (dashed line), with addition of $3 \mathrm{mM}$ $\mathrm{Ca}^{2+}$ (dash-dot-dot-dot line).

The temperature dependence of the first derivative of the CD signal (upper panel) and the absorbance (lower panel) at $222 \mathrm{~nm}$ are shown in Figure 10a for full-length HsCen-2 in a low ionic strength buffer. These data show a single broad temperature transition at $\sim 53{ }^{\circ} \mathrm{C}$ for apoHsCen-2, whereas two transitions are observed in the $\mathrm{Ca}^{2+}$-saturated state, one quite broad at $\sim 24{ }^{\circ} \mathrm{C}$ and another at $\sim 77^{\circ} \mathrm{C}$ with a higher degree of cooperativity. The absorbance of both buffer and apo-protein gradually increase with increased temperature, an expected "normal" temperature dependence. However, the $\mathrm{Ca}^{2+}$-saturated HsCen-2 shows an increase of turbidity in the temperature range $10-30{ }^{\circ} \mathrm{C}$ from which we infer the formation of aggregates. Higher temperatures appear to lead to dissolution of these aggregates as the absorbance is again close to that seen in apo-state. In Figure $10 \mathrm{~b}$ are shown the results of similar measurements for $\Delta 25$ HsCen-2. This truncated protein shows a single temperature transition in the apo-state just as with the full-length protein, though at a lower temperature of $\sim 35^{\circ} \mathrm{C}$. Only very minor differences are found for the $\mathrm{Mg}^{2+}$-saturated state. Whereas in the $\mathrm{Ca}^{2+}$-saturated state of $\Delta 25 \mathrm{HsCen}-2$, two temperature transitions are again observed at $\sim 26^{\circ} \mathrm{C}$ and $\sim 86{ }^{\circ} \mathrm{C}$, slightly higher values than found for the native protein. Also noted, no aggregation is observed with $\Delta 25 \mathrm{HsCen}-2$ in complex with $\mathrm{Ca}^{2+}$ at any temperature examined. Figure 10c shows the effects of higher ionic strength $(100 \mathrm{mM} \mathrm{KCl})$ buffer on native HsCen-2. The mid-point temperature transition for the apo-state and the higher-temperature transition of the $\mathrm{Ca}^{2+}$-loaded state were increased $3-4{ }^{\circ} \mathrm{C}$. Interestingly, the mid-point of the lower-temperature transition for the $\mathrm{Ca}^{2+}$-loaded protein increased by $23{ }^{\circ} \mathrm{C}$ compared to that seen in Figure 10a (low ionic strength buffer). The more physiological ionic strength also prevented the macroscopic aggregate formation seen without salt. The temperature dependence of these $\mathrm{CD}$ and absorbance data in Figure 10 are $70-90 \%$ reversible in repeated cycles after rapid cooling from $91{ }^{\circ} \mathrm{C}$ back to $0{ }^{\circ} \mathrm{C}$.

This is the first examination using $\mu$ ESI-MS of the metal binding to HsCen-2. As shown in Figure 2 and Figure 8, the $\mu$ ESI-MS spectra of both the HsCen-2 and $\Delta 25$ HsCen-2 show two charge-state distributions that respond differently to added cations (Figures 3-6 for full-length HsCen-2 and for $\Delta 25 \mathrm{HsCen}-2$, those data are not shown). The two charge-state distributions may represent the following: conformation 1: A disordered state that when electrosprayed, yields more charging due to exposure of amino acid side chains, and less cation binding due to disordered binding structures. Indeed, Prakash and Mazumdar [81] showed that the higher charged states in positive ion $\mu$ ESI-MS are a consequence of unfolding, affording a greater number of surface basic groups. Denatured calmodulin also sprays with higher charge states than does the wild type [82]. Others suggest, however, that the higher charged states correlate only with surface area and not the number of exposed side chains [83]. Conformation 2: A folded, native state of the protein with normally folded EF-hand sequences. A similar bimodal charge-state distribution in $10 \mathrm{mM}$ ammonium acetate was described for an ESI-MS of calmodulin [82]. Nevertheless, HsCen-2 in conformation 2 continues to add calcium until the apo-protein becomes a minor component of the total. The highest and next highest affinity calcium binding sites of the HsCen-2 took up most of the $\mathrm{Ca}^{2+}$, suggesting that intact EF-hands and intact protein structure could account for the extensive calcium loading of HsCen-2. Conformation 1, by contrast, loaded calcium to a much lesser extent.

As shown in Figure 3, we found that HsCen-2 binds one $\mathrm{Ca}^{2+}$ with high affinity. We also found that the protein has three low affinity $\mathrm{Ca}^{2+}$-binding sites. We know from studies of other proteins that the changes in 
charge-state distributions correlate with changes in $\mathrm{CD}$ and fluorescence $[24,25,27-29,32]$. The binding of $\mathrm{Ca}^{2+}$ to HsCen-2 does not result in a significant change in the charge state distribution of the protein, suggesting little change in secondary structure, a finding confirmed by far-UV spectroscopy (Figure 9 and Table 1). In addition, as demonstrated in Figure 4, we found that HsCen-2 binds one $\mathrm{Mg}^{2+}$ with high affinity and three $\mathrm{Mg}^{2+}$ with lower affinity. Furthermore, our data demonstrate that the high affinity $\mathrm{Ca}^{2+}$-binding site is different than the high affinity $\mathrm{Mg}^{2+}$-binding site (Figures 5 and 6). The binding of $\mathrm{Mg}^{2+}$ to HsCen-2 does not cause a significant change in the charge state distribution of the electrospray protein, indicating a lack of change in tertiary and secondary structure that was confirmed in near- and far-UV spectroscopy experiments (Figure 9 and Table 1).

We are able to demonstrate that $\mathrm{Ca}^{2+}$ readily competes for $\mathrm{Mg}^{2+}$-binding sites in the $\mathrm{Mg}^{2+}$-saturated protein. The same is true of $\mathrm{Mg}^{2+}$, which is capable of displacing $\mathrm{Ca}^{2+}$ from the $\mathrm{Ca}^{2+}$-saturated protein, although $\mathrm{Ca}^{2+}$ appears to be more efficient in displacing $\mathrm{Mg}^{2+}$ than the opposite. $\Delta 25 \mathrm{HsCen}-2$ has metal-binding properties that are similar to those of full-length HsCen-2.

The binding of $\mathrm{Ca}^{2+}$ and $\mathrm{Mg}^{2+}$ to HsCen-2 appears to be similar to that observed for DREAM/calsenilin [25], but the binding is distinct from that of calbindin $\mathrm{D}_{28 \mathrm{~K}}$ [23] which, from $\mu$ ESI-MS results, rapidly loads to give the $4 \mathrm{Ca}^{2+}$-calbindin $\mathrm{D}_{28 \mathrm{~K}}$ form of the protein.

Our results are in contrast to those of Durussel et al., who observed by gel-filtration methods that HsCen-2 exists as a $1.5 \mathrm{Ca}^{2+} / \mathrm{HsCen}-2$ dimer and $2 \mathrm{Ca}^{2+} / \mathrm{HsCen}-2$ dimer, and by flow dialysis, a $4 \mathrm{Ca}^{2+} / \mathrm{HsCen}-2$ dimer complexed to two melittin peptides [19]. The high affinity $\mathrm{Ca}^{2+}$ binding site was likely site IV in each monomer [19]. Additional $\mathrm{Ca}^{2+}$ binding sites were of low affinity. Other centrins for which $\mathrm{Ca}^{2+}$-binding was reported include Chlamydomonas centrin, which binds four moles of calcium $/ \mathrm{mol}$ protein [22]. No data were previously reported concerning the $\mathrm{Mg}^{2+}$-binding stoichiometry of HsCen-2 or Chlamydomonas centrin other than to note that the uptake of $\mathrm{Mg}^{2+}$ reduces the affinity of calcium in both proteins $[19,80]$.

Even though there are small increases in the amplitude of the far-UV CD spectra (Figure 9), the secondary structures of $\mathrm{HsCen}-2$ in apo-, $\mathrm{Mg}^{2+}$ - and $\mathrm{Ca}^{2+}$-saturated states turn out to be practically the same (Table 1). The only difference is a $3 \pm 2 \%$ increase in total helicity upon $\mathrm{Ca}^{2+}$ but not $\mathrm{Mg}^{2+}$-binding. This increase in helicity also has to be attributed to folding of $\mathrm{N}$-terminal domain upon $\mathrm{Ca}^{2+}$ addition. The near-UV CD spectra do not show any signal from the only Tyr 172 of HsCen-2; the sharp negative bands in the spectral range $250-270 \mathrm{~nm}$ belong to the 10 phenylalanine residues. These results imply that the Cterminus of HsCen-2 (side-chain of Tyr) is free and is not fixed in the protein tertiary structure. The near-UV CD spectra demonstrate that there are no changes in tertiary structure upon $\mathrm{Mg}^{2+}$-binding. Analysis of spectral changes upon $\mathrm{Ca}^{2+}$ binding shows changes in the asymmetry of phenylalanine microenvironments and, there- fore, indicates some change in protein tertiary structure. These changes in the near-UV CD spectra can also be ascribed to the appearance of induced optical activity of phenylalanine residues in the $\mathrm{N}$-terminal domain when it is stabilized and folded as a result of $\mathrm{Ca}^{2+}$ addition.

Apo-HsCen-2, in a buffer with low ionic strength, has one very broad temperature transition at $\sim 53^{\circ} \mathrm{C}$. In the $\mathrm{Ca}^{+2}$-saturated state it has two peaks at $\sim 24$ (broad) and $\sim 77^{\circ} \mathrm{C}$ (much sharper and more cooperative than the first one). An increase in temperature in the range $10-30{ }^{\circ} \mathrm{C}$, leads to reversible aggregation of protein in the $\mathrm{Ca}^{2+}$ loaded state. For the protein missing the $\mathrm{N}$-terminal 25 residues and in a low ionic strength buffer, an increase of buffer ionic strength does not cause aggregation of the protein. A more stable structural domain, with a midpoint temperature of denaturation at $\sim 50-56{ }^{\circ} \mathrm{C}$ in the apostate, probably results from denaturation of the C-terminal part of HsCen-2 [19], which is likely to have within it the high affinity $\mathrm{Ca}^{2+}$-binding site. On the basis of these data, it is unlikely that apo-HsCen-2 would crystallize at $\sim 0{ }^{\circ} \mathrm{C}$, because the $\mathrm{N}$-terminal half of molecule would be disordered at this temperature.

Small increases in the amplitude of the far- or near-UV $\mathrm{CD}$ spectra upon $\mathrm{Ca}^{2+}$ binding by $\mathrm{HsCen}-2$ indicate small changes in the secondary structure, and some change in the tertiary structure of the protein. This can be attributed to changes in protein stability (rigidity). One structural and functional domain of HsCen-2 in the apo-state is melted at $<0{ }^{\circ} \mathrm{C}$; a second one has the midpoint of heat denaturation at $\sim 53{ }^{\circ} \mathrm{C}$. The first domain is stabilized by $\mathrm{Ca}^{2+}$-binding and the midpoint of heat denaturation is shifted to higher temperatures $\left(24-47^{\circ} \mathrm{C}\right)$. The midpoint of heat denaturation for the second domain is shifted to $77-86^{\circ} \mathrm{C}$ with an increase in cooperativity of denaturation.

Overall, the far- and near-CD studies indicate little $\mathrm{Ca}^{2+}$ - or $\mathrm{Mg}^{2+}$-induced structural changes while the temperature denaturation studies show $\mathrm{Ca}^{2+}$-specific effects. Other indications that there are no large structural changes on metal binding include the lack of changes in both the ESI charge-state distributions upon metal addition (Figure 2; see Results) and in nondenaturing polyacrylamide gels of apo-HsCen-2 when calcium or magnesium was added in a 4:1 $\mathrm{M}$ ratio; no significant changes in electrophoretic mobility of HsCen-2 occurred (data not shown). In addition, in H/D exchange studies, the deuterium uptake of the apo-HsCen-2 is similar to that of holo (20-fold excess $\mathrm{Ca}^{2+}$ ) studied as a function of time, suggesting that the conformational change of the protein upon binding to $\mathrm{Ca}^{2+}$ is small [84].

\section{Conclusions}

Our investigations have, for the first time, defined that HsCen-2 binds one calcium with relatively high affinity, a second with significantly lower affinity and two more with lower affinity yet. A similar pattern pertains for $\mathrm{Mg}^{2+}$ binding, although the binding sites for $\mathrm{Ca}^{2+}$ are relatively more specific. Removal of the N-terminal 25 
residues affords a HsCen-2 that remains soluble even after addition of $\mathrm{Ca}^{2+}$. Increasing the ionic strength does not induce full length HsCen-2 to assemble into an aggregate insoluble complex upon addition of $\mathrm{Ca}^{2+}$. This lack of aggregation may promote efforts to crystallize the $\mathrm{Ca}^{2+}$-bound forms of HsCen-2. Addition of $\mathrm{Ca}^{2+}$, but not $\mathrm{Mg}^{2+}$, to HsCen-2 stabilizes the protein.

\section{Acknowledgments}

Supported by NIH grants DK58546 and DK65830 (to RK) and NIH grant P41RR00954 (to MLG).

\section{References}

1. Baron, A. T.; Salisbury, J. L. Identification and localization of a novel, cytoskeletal, centrosome-associated protein in PtK2 cells. J. Cell. Biol. 1988, 107(6 Pt 2), 2669-2678.

2. Sanders, M. A.; Salisbury, J. L. Centrin-mediated microtubule severing during flagellar excision in Chlamydomonas reinhardtii. J. Cell. Biol. 1989, 108(5), 1751-1760

3. Coling, D. E.; Salisbury, J. L. Characterization of the calcium-binding contractile protein centrin from Tetraselmis striata (Pleurastrophyceae). J. Protozool. 1992, 39(3), 385-391.

4. Salisbury, J. L. Centrin, centrosomes, and mitotic spindle poles. Curr. Opin. Cell Biol. 1995, 7(1), 39-45.

5. Salisbury, J. L.; Suino, K. M.; Busby, R.; Springett, M. Centrin-2 is required for centriole duplication in mammalian cells. Curr. Biol. 2002, 12(15), 1287-1292.

6. Salisbury, J. L.; Baron, A.; Surek, B.; Melkonian, M. Striated flagellar roots: Isolation and partial characterization of a calcium-modulated contractile organelle. J. Cell Biol. 1984, 99(3), 962-970.

7. Huang, B.; Mengersen, A.; Lee, V. D. Molecular cloning of cDNA for caltractin, a basal body-associated $\mathrm{Ca} 2+$ binding protein: Homology in its protein sequence with calmodulin and the yeast CDC31 gene product. J. Cell Biol. 1988, 107(1), 133-140.

8. Gonda, K.; Yoshida, A.; Oami, K.; Takahashi, M. Centrin is essential for the activity of the ciliary reversal-coupled voltage-gated Ca2+ channels. Biochem. Biophys. Res. Commun. 2004, 323(3), 891-897.

9. Keller, L. C.; Romijn, E. P.; Zamora, I.; Yates, J. R., III; Marshall, W. F. Proteomic analysis of isolated chlamydomonas centrioles reveals orthologs of ciliary-disease genes. Curr. Biol. 2005, 15(12), 1090-1098.

10. Giessl, A.; Pulvermuller, A.; Trojan, P.; Park, J. H.; Choe, H. W.; Ernst, O. P.; Hofmann, K. P.; Wolfrum, U. Differential expression and interaction with the visual G-protein transducin of centrin isoforms in mammalian photoreceptor cells. J. Biol. Chem. 2004, 279(49), 5147251481.

11. Wolfrum, U.; Giessl, A.; Pulvermuller, A. Centrins, a novel group of $\mathrm{Ca} 2+$ binding proteins in vertebrate photoreceptor cells. Adv. Exp. Med. Biol. 2002, 514, 155-178.

12. Wolfrum, U.; Salisbury, J. L. Expression of centrin isoforms in the mammalian retina. Exp. Cell Res. 1998, 242(1), 10-17.

13. Kilmartin, J. V. Sfilp has conserved centrin-binding sites and an essential function in budding yeast spindle pole body duplication. J. Cell Biol. 2003, 162(7), 1211-1221.

14. Salisbury, J. L. Centrosomes: Sfi1p and centrin unravel a structural riddle. Curr. Biol. 2004, 14(1), R27-R29.

15. Araki, M.; Masutani, C.; Takemura, M.; Uchida, A.; Sugasawa, K.; Kondoh, J.; Ohkuma, Y.; Hanaoka, F. Centrosome protein centrin 2 /caltractin 1 is part of the xeroderma pigmentosum group $\mathrm{C}$ complex that initiates global genome nucleotide excision repair. J. Biol. Chem. 2001, 276(22), 18665-18672.

16. Popescu, A.; Miron, S.; Blouquit, Y.; Duchambon, P.; Christova, P.; Craescu, C. T. Xeroderma pigmentosum group $\mathrm{C}$ protein possesses a high affinity binding site to human centrin 2 and calmodulin. J. Biol. Chem. 2003, 278(41), 40252-40261.

17. Baron, A. T.; Greenwood, T. M.; Bazinet, C. W.; Salisbury, J. L. Centrin is a component of the pericentriolar lattice. Biol. Cell 1992, 76(3), 383-388.

18. Paoletti, A.; Moudjou, M.; Paintrand, M.; Salisbury, J. L.; Bornens, M. Most of centrin in animal cells is not centrosome-associated and centrosomal centrin is confined to the distal lumen of centrioles. J. Cell Sci. 1996, 109(Pt 13), 3089-3102.

19. Durussel, I.; Blouquit, Y.; Middendorp, S.; Craescu, C. T.; Cox, J. A. Cation- and peptide-binding properties of human centrin 2. FEBS Lett. 2000, 472(2/3), 208-212.

20. Matei, E.; Miron, S.; Blouquit, Y.; Duchambon, P.; Durussel, I.; Cox, J. A.; Craescu, C. T. C-terminal half of human centrin 2 behaves like a regulatory EF-hand domain. Biochemistry 2003, 42(6), 1439-1450.

21. Tourbez, M.; Firanescu, C.; Yang, A.; Unipan, L.; Duchambon, P.; Blouquit, Y.; Craescu, C. T. Calcium-dependent self-assembly of human centrin 2. J. Biol. Chem. 2004, 279(46), 47672-47680.
22. Veeraraghavan, S.; Fagan, P. A.; Hu, H.; Lee, V.; Harper, J. F.; Huang, B.; Chazin, W. J. Structural independence of the two EF-hand domains of caltractin. J. Biol. Chem. 2002, 277(32), 28564-28571.

23. Kumar, R.; Hunziker, W.; Gross, M.; Naylor, S. Londowski, J. M. Schaefer, J. The highly efficient production of full-length and mutant rat brain calcium-binding proteins (calbindins-D28K) in a bacterial expression system. Arch. Biochem. Biophys. 1994, 308(1), 311-317.

24. Craig, T. A.; Veenstra, T. D.; Naylor, S.; Tomlinson, A. J.; Johnson, K. L.; Macura, S.; Juranic, N.; Kumar, R. Zinc binding properties of the DNA binding domain of the 1,25-dihydroxyvitamin D3 receptor. Biochemistry 1997, 36(34), 10482-10491.

25. Veenstra, T. D.; Johnson, K. L.; Tomlinson, A. J.; Naylor, S.; Kumar, R. Determination of calcium-binding sites in rat brain calbindin D28K by electrospray ionization mass spectrometry. Biochemistry 1997, 36(12), 3535-3542.

26. Veenstra, T. D.; Tomlinson, A. J.; Benson, L.; Kumar, R.; Naylor, S. Low temperature aqueous electrospray ionization mass spectrometry of noncovalent complexes. J. Am. Soc. Mass Spectrom. 1998, 9(6), 580-584.

27. Veenstra, T. D.; Johnson, K. L.; Tomlinson, A. J.; Craig, T. A.; Kumar, R.; Naylor, S. Zinc-induced conformational changes in the DNA-binding domain of the vitamin $D$ receptor determined by electrospray ionization mass spectrometry. J. Am. Soc. Mass Spectrom. 1998, 9(1), 8-14.

28. Veenstra, T. D.; Benson, L. M.; Craig, T. A.; Tomlinson, A. J.; Kumar, R.; Naylor, S. Metal mediated sterol receptor-DNA complex association and dissociation determined by electrospray ionization mass spectrometry. Nat. Biotechnol. 1998, 16(3), 262-266.

29. Craig, T. A.; Benson, L. M.; Tomlinson, A. J.; Veenstra, T. D.; Naylor, S.; Kumar, R. Analysis of transcription complexes and effects of ligands by microelectrospray ionization mass spectrometry. Nat. Biotechnol. 1999, 17(12), 1214-1218.

30. Johnson, K. L.; Veenstra, T. D.; Londowski, J. M.; Tomlinson, A. J.; Kumar, R.; Naylor, S. On-line sample clean-up and chromatography coupled with electrospray ionization mass spectrometry to characterize the primary sequence and disulfide bond content of recombinant calcium binding proteins. Biomed. Chromatogr. 1999, 13(1), 37-45.

31. Craig, T. A.; Benson, L. M.; Naylor, S.; Kumar, R. Modulation effects of zinc on the formation of vitamin $D$ receptor and retinoid $X$ receptor $\alpha$-DNA transcription complexes: Analysis by microelectrospray mass spectrometry. Rapid Commun. Mass Spectrom. 2001, 15(12), 1011-1016.

32. Craig, T. A.; Benson, L. M.; Venyaminov, S. Y.; Klimtchuk, E. S.; Bajzer, Z.; Prendergast, F. G.; Naylor, S.; Kumar, R. The metal-binding properties of DREAM: evidence for calcium-mediated changes in DREAM structure. J. Biol. Chem. 2002, 277(13), 10955-10966.

33. Loo, J. A. Studying noncovalent protein complexes by electrospray ionization mass spectrometry. Mass Spectrom. Rev. 1997, 16(1), 1-23.

34. van Berkel, W. J.; van den Heuvel, R. H.; Versluis, C.; Heck, A. J. Detection of intact megaDalton protein assemblies of vanillyl-alcohol oxidase by mass spectrometry. Protein Sci. 2000, 9(3), 435-439.

35. Zhang, Z.; Krutchinsky, A.; Endicott, S.; Realini, C.; Rechsteiner, M.; Standing, K. G. Proteasome activator 11S REG or PA28: Recombinant REG $\alpha$ /REG $\beta$ hetero-oligomers are heptamers. Biochemistry 1999, 38(17), 5651-5658.

36. Rostom, A. A.; Fucini, P.; Benjamin, D. R.; Juenemann, R.; Nierhaus, K. H.; Hartl, F. U.; Dobson, C. M.; Robinson, C. V. Detection and selective dissociation of intact ribosomes in a mass spectrometer. Proc. Natl. Acad. Sci. U.S.A. 2000, 97(10), 5185-5190.

37. Hernandez, H.; Robinson, C. V. Dynamic protein complexes: Insights from mass spectrometry. J. Biol. Chem. 2001, 276(50), 46685-46688.

38. Tito, M. A.; Miller, J.; Walker, N.; Griffin, K. F.; Williamson, E. D.; Despeyroux-Hill, D.; Titball, R. W.; Robinson, C. W. Probing molecular interactions in intact antibody: Antigen complexes, an electrospray time-of-flight mass spectrometry approach. Biophys. J. 2001, 81(6), 3503-3509.

39. Elviri, L.; Zagnoni, I.; Careri, M.; Cavazzini, D.; Rossi, G. L. Noncovalent binding of endogenous ligands to recombinant cellular retinolbinding proteins studied by mass spectrometric techniques. Rapid Commun. Mass Spectrom. 2001, 15(22), 2186-2192.

40. Potier, N.; Rogniaux, H.; Chevreux, G.; Van Dorsselaer, A. Ligand-metal ion binding to proteins: Investigation by ESI mass spectrometry. Methods Enzymol. 2005, 402, 361-389.

41. Greig, M. J.; Gaus, H.; Cummins, L. L.; Sasmor, H.; Griffey, R. H. Measurement of macromolecular binding using electrospray mass spectrometry. Determination of dissociation constants for oligonucleotide: Serum albumin complexes. J. Am. Chem. Soc. 1995, 117, 1076510766.

42. Sannes-Lowry, K. A.; Mei, H. Y.; Loo, J. A. Studying aminoglycoside antibiotic binding to HIV-1 TAR RNA by electrospray ionization mass spectrometry. Int. I. Mass Spectrom. 1999, 193, 115-122.

43. Loo, J. A.; Edmonds, C. G.; Smith, R. D. Tandem mass spectrometry of very large molecules: serum albumin sequence information from multiply charged ions formed by electrospray ionization. Anal. Chem. 1991, 63(21), 2488-2499.

44. Chowdhuray, S. K.; Katta, V.; Chait, B. T. Probing conformational changes in proteins by mass spectrometry. J. Am. Chem. Soc. 1990, 112, 9012-9013.

45. Mirza, U. A.; Cohen, S. L.; Chait, B. T. Heat-induced conformational changes in proteins studied by electrospray ionization mass spectrometry. Anal. Chem. 1993, 65(1), 1-6.

46. Mohimen, A.; Dobo, A.; Hoerner, J. K.; Kaltashov, I. A. A chemometric approach to detection and characterization of multiple protein conform- 
ers in solution using electrospray ionization mass spectrometry. Anal. Chem. 2003, 75(16), 4139-4147.

47. Ray, S. S.; Singh, S. K.; Balaram, P. An electrospray ionization mass spectrometry investigation of 1-anilino-8-naphthalene-sulfonate (ANS) binding to proteins. J. Am. Soc. Mass Spectrom. 2001, 12, 428-438.

48. Cohen, S. A.; Bidlingmeyer, B. A.; Tarvin, T. L. PITC derivatives in amino acid analysis. Nature 1986, 320(6064), 769-770.

49. Bidlingmeyer, B. A.; Cohen, S. A.; Tarvin, T. L. Rapid analysis of amino acids using pre-column derivatization. J. Chromatogr. 1984, 336(1), 93-104.

50. Hunkapiller, M. W.; Hewick, R. M.; Dreyer, W. J.; Hood, L. E. Highsensitivity sequencing with a gas-phase sequenator. Methods Enzymol. 1983, 91, 399-413.

51. Hewick, R. M.; Hunkapiller, M. W.; Hood, L. E.; Dreyer, W. J. A gas-liquid solid phase peptide and protein sequenator. J. Biol. Chem. 1981, 256(15), 7990-7997.

52. Sanger, F.; Nicklen, S.; Coulson, A. R. DNA sequencing with chainterminating inhibitors, 1977. Biotechnology 1992, 24, 104-108.

53. Sanger, F.; Nicklen, S.; Coulson. A. R. DNA sequencing with chainterminating inhibitors. Proc. Natl. Acad. Sci. U.S.A. 1977, 74(12), 54635467.

54. Caruthers, M. H.; Beaucage, S. L.; Efcavitch, J. W.; Fisher, E. F.; Matteucci, M. D.; Stabinsky, Y. New chemical methods for synthesizing polynucleotides. Nucleic Acids Symp. Ser. 1980, 7, 215-223.

55. Matteucci, M. D.; Caruthers, M. H. Synthesis of deoxyoligonucleotides on a polymer support, 1981. Biotechnology 1992, 24, 92-98.

56. Bradford, M. M. A rapid and sensitive method for the quantitation of microgram quantities of protein utilizing the principle of protein-dye binding. Anal. Biochem. 1976, 72, 248-254.

57. Craig, T. A.; Kumar, R. Synthesis and purification of soluble ligand binding domain of the human vitamin D3 receptor. Biochem. Biophys. Res. Commun. 1996, 218(3), 902-907.

58. Berndt, T.; Craig, T. A.; Bowe, A. E.; Vassiliadis, J.; Reczek, D.; Finnegan, R.; Jan DeBeur, S. M.; Schiavi, S. C.; Kumar, R. Secreted frizzled-related protein 4 is a potent tumor-derived phosphaturic agent. J. Clin. Invest. 2003, 112(5), 785-794.

59. Veenstra, T. D.; Gross, M. D.; Hunziker, W.; Kumar, R. Identification of metal-binding sites in rat brain calcium-binding protein. J. Biol. Chem. 1995, 270(51), 30353-30358.

60. Gross, M. D.; Kumar, R.; Hunziker, W. Expression in Escherichia coli of full-length and mutant rat brain calbindin D28. Comparison with the purified native protein. J. Biol. Chem. 1988, 263(28), 14426-14432.

61. Sreerama, N.; Woody, R. W. Estimation of protein secondary structure from circular dichroism spectra: comparison of CONTIN, SELCON, and CDSSTR methods with an expanded reference set. Anal. Biochem. 2000, 287(2), 252-260

62. Sreerama, N.; Venyaminov, S. Y.; Woody, R. W. Estimation of the number of alpha-helical and beta-strand segments in proteins using circular dichroism spectroscopy. Protein Sci. 1999, 8(2), 370-380.

63. Provencher, S. W.; Glockner, J. Estimation of globular protein secondary structure from circular dichroism. Biochemistry 1981, 20(1), 33-37.

64. van Stokkum, I. H.; Spoelder, H. J.; Bloemendal, M.; van Grondelle, R.; Groen, F. C. Estimation of protein secondary structure and error analysis from circular dichroism spectra. Anal. Biochem. 1990, 191(1), 110-118.

65. Johnson, W. C. Analyzing protein circular dichroism spectra for accurate secondary structures. Proteins 1999, 35(3), 307-312.

66. Venyaminov, S.; Vassilenko, K. S. Determination of protein tertiary structure class from circular dichroism spectra. Anal. Biochem. 1994, 222(1), 176-184
67. Sreerama, N.; Venyaminov, S. Y.; Woody, R. W. Estimation of protein secondary structure from circular dichroism spectra: Inclusion of denatured proteins with native proteins in the analysis. Anal. Biochem. 2000, 287(2), 243-251.

68. Mihaly E. J. Numerical values of the adsorbances of the aromatic amino acids in acid, neutral and alkaline solutions. Chem. Eng. Data 1968, 13, 179-182.

69. Gill, S. C.; von Hippel, P. H. Calculation of protein extinction coefficients from amino acid sequence data. Anal. Biochem. 1989, 182(2), 319-326.

70. Mach, H.; Middaugh, C. R.; Lewis, R. V. Statistical determination of the average values of the extinction coefficients of tryptophan and tyrosine in native proteins. Anal. Biochem. 1992, 200(1), 74-80.

71. Pace, C. N.; Vajdos, F.; Fee, L.; Grimsley, G.; Gray, T. How to measure and predict the molar absorption coefficient of a protein. Protein Sci. 1995, 4(11), 2411-2423.

72. Persechini, A.; Moncrief, N. D.; Kretsinger, R. H. The EF-hand family of calcium-modulated proteins. Trends Neurosci. 1989, 12(11), 462-467.

73. Moncrief, N. D.; Kretsinger, R. H.; Goodman, M. Evolution of EF-hand calcium-modulated proteins. I. Relationships based on amino acid sequences. J. Mol. Evol. 1990, 30(6), 522-562.

74. Nakayama, S.; Moncrief, N. D.; Kretsinger, R. H. Evolution of EF-hand calcium-modulated proteins. II. Domains of several subfamilies have diverse evolutionary histories. J. Mol. Evol. 1992, 34(5), 416-448.

75. Nakayama, S.; Kretsinger, R. H. Evolution of EF-hand calciummodulated proteins. III. Exon sequences confirm most dendrograms based on protein sequences: Calmodulin dendrograms show significant lack of parallelism. J. Mol. Evol. 1993, 36(5), 458-476.

76. Kretsinger, R. H.; Nakayama, S. Evolution of EF-hand calciummodulated proteins. IV. Exon shuffling did. not determine the domain compositions of EF-hand proteins. J. Mol. Evol. 1993, 36(5), 477-488.

77. Nakayama, S.; Kretsinger, R. H. Evolution of the EF-hand family of proteins. Annu. Rev. Biophys. Biomol. Struct. 1994, 23, 473-507.

78. Kawasaki, H.; Nakayama, S.; Kretsinger, R. H. Classification and evolution of EF-hand proteins. Biometals 1998, 11(4), 277-295.

79. Wiech, H.; Geier, B. M.; Paschke, T.; Spang, A.; Grein, K.; Steinkotter, J.; Melkonian, M.; Schiebel, E. Characterization of green alga, yeast, and human centrins. Specific subdomain features determine functional diversity. J. Biol. Chem. 1996, 271(37), 22453-22461.

80. Hu, H.; Sheehan, J. H.; Chazin, W. J. The mode of action of centrin. Binding of $\mathrm{Ca} 2+$ and a peptide fragment of Kar1p to the C-terminal domain. J. Biol. Chem. 2004, 279(49), 50895-50903.

81. Prakash, H.; Mazumdar, S. Direct correlation of the crystal structure of proteins with the maximum positive and negative charge states of gaseous protein ions produced by electrospray ionization. J. Am. Soc. Mass Spectrom. 2005, 16(9), 1409-1421.

82. Lafitte, D.; Heck, A. J.; Hill, T. J.; Jumel, K.; Harding, S. E.; Derrick, P. J. Evidence of noncovalent dimerization of calmodulin. Eur. J. Biochem. 1999, 261(1), 337-344

83. Hautreux, M.; Hue, N.; Alexis, Du Fou de Kerdaniel, A.; Zahir, A.; Malec, V.; Laprévote, O. Under nondenaturing solvent conditions, the mean charge state of a multiply charged protein ion formed by electrospray is linearly correlated with the macromolecular surface. Int. J. Mass Spectrom. 2004, 231, 131-137.

84. Sperry, J.; Gross, M.; Kumar, R. Calcium binding properties of human centrin 2. Proceedings of the 53rd ASMS Conference on Mass Spectrometry and Allied Topics; San Antonio, TX, June, 2005. 